\title{
Mielopatía cervical: análisis retrospectivo de los resultados quirúrgicos de 54 pacientes tratados mediante discectomía y fusión intersomática por vía anterior
}

\author{
R. Martín; J.R. Carda; F. Montiaga; J.I. Pinto; F. Sanz; B. Paternina; F. Trigueros; J.M. Izquierdo y A. Vázquez- \\ Barquero
}

Servicio de Neurocirugía. Hospital Universitario Marqués de Valdecilla. Santander.

Resumen

Pacientes y métodos. Se analiza de forma retrospectiva el resultado clínico de una serie consecutiva de 53 pacientes afectos de mielopatía o mielorradiculopatía cervical que fueron intervenidos mediante discectomía cervical y artrodesis por vía anterior utilizando la técnica de Cloward, con un seguimiento medio de 3 años.

Resultados. El $64.2 \%$ de los pacientes se benefició del tratamiento quirúrgico, mejorando en uno o más grados en la escala de Nurick. No se produjo mortalidad, aunque apareció deterioro neurológico en el $9.4 \%$ de los casos. Se objetivó una correcta fusión de los segmentos intervenidos en el $\mathbf{9 2 . 5 \%}$ de las cirugías, desarrollando un $\mathbf{9 . 4 \%}$ de los enfermos cifosis postquirúrgica. En el análisis multivariante se identificaron como factores relacionados con el resultado clínico: edad $(p=0.008)$, presencia de factores de riesgo vascular $(p=0.031)$, duración de la clínica $(\mathrm{p}=\mathbf{0 . 0 0 2})$, estado neurológico prequirúrgico $(\mathrm{p}<0.001)$, diagnóstico neurorradiológico $(p=0.014)$, presencia de hiperseñal intramedular en las secuencias de resonancia magnética potenciadas en $\mathrm{T} 2$ $(p=0.008)$, alteración de la conducción medular en el estudio neurofisiológico $(p=0.004)$ y desarrollo de complicaciones quirúrgicas $(\mathrm{p}=\mathbf{0 . 0 1 2})$.

Conclusiones. Dadas las discrepancias existentes en la actualidad, la optimización del tratamiento de estos pacientes pasa por una valoración individualizada, y por la realización de estudios prospectivos y randomizados que respondan a las preguntas cuándo y cómo operar.

PALABRAS CLAVE: Artrodesis intersomática. Discectomía cervical. Hernia discal cervical. Mielopatía cervical. Radiculopatía cervical.

Cervical myelopathy: Retrospective analysis of surgical results in $\mathbf{5 3}$ cases treated by anterior cervical discectomy and interbody fusion

Recibido: 1-10-03. Aceptado: 12-11-03
Abstract

Patients and methods. We perform a retrospective analysis of clinical results in 53 consecutive patients surgically treated for cervical myelopathy or myeloradiculopathy with anterior cervical discectomy and interbody fusion by means of the Cloward procedure.

Results. $64.2 \%$ of the patients had good outcome as measured by the improvement in one or more grades in the Nurick's scale. No mortality related to the surgical procedure was noted, although $9.4 \%$ of the cases suffered neurological deterioration. Correct fusion was achieved in $92.5 \%$ of the patients, with a rate of post-surgical kyphosis of $\mathbf{9 . 4 \%}$. Multivariate analysis identified as factors related to the clinical outcome: age $(p=0.008)$, vascular risk factors $(p=0.031)$, duration of symptoms $(\mathrm{p}=\mathbf{0 . 0 0 2})$, pre-surgical neurological status $(p<0.001)$, neuroradiological diagnosis $(p=0.014)$, intramedullary high signal intensity changes in $\mathrm{T} 2$-weighted images $(p=0.008)$, prolongation of the central somatosensory or motor conduction times $(p=0.004)$ and neurologic complications $(\mathrm{p}=\mathbf{0 . 0 1 2})$

Conclusions. Treatment optimisation of the patient suffering cervical spondylotic myelopathy requires individualised evaluation. Prospective randomised studies are needed to answer the questions when and how to operate.

KEY WORDS: Cervical discectomy. Cervical disc herniation. Cervical myelopathy. Interbody fusion.

\section{Introducción}

La mielopatía cervical espondilótica es una patología frecuente, que afecta fundamentalmente a varones por encima de los 50 años y representa la causa más frecuente de disfunción del cordón espinal por encima de los 55 años $^{57}$. En el estudio prospectivo de Moore y cols, la mielopatía cervical espondilótica daba cuenta de una cuarta parte de las paraparesias y tetraparesias de origen no traumático ${ }^{47}$. 


\section{Tabla 1}

\section{Escala de Nurick}
Grado 0
Signos y síntomas radiculares. Sin evidencia de afectación del cordón medular
Grado 1
Signos de afectación del cordón medular, pero sin alteración de la marcha
Grado 2
Dificultad de la marcha leve que no interfiere en la actividad laboral
Grado 3
Dificultad de la marcha que interfiere en la actividad laboral
Grado 4
Necesidad de ayuda para caminar (andador)
Grado 5
Silla de ruedas o encamado

La historia natural de esta enfermedad parece alternar episodios de deterioro neurológico progresivo, más o menos rápido, con largos periodos de estabilización, motivo por el que desde hace más de medio siglo se indica la descompresión quirúrgica, en un intento de mejorar su evolución, con resultados dispares ${ }^{7,53,57}$. Las controversias acerca del abordaje quirúrgico de la mielopatía cervical espondilótica aún no se han resuelto y algunos autores prefieren los abordajes anteriores, mientras que otros se decantan por los posteriores $7,14,21,39,59$. De hecho, la evidencia científica de la superioridad del tratamiento quirúrgico sobre el conservador, si existente, es débil ${ }^{18,33,58}$ y los estudios que han comparado los resultados clínicos de los diferentes procedimientos por vía anterior y por vía posterior no resultan totalmente definitorios ${ }^{14,16,27,28,31,71,75}$.

Hasta que se alcance un consenso acerca de cuándo y cómo intervenir a estos pacientes -al que probablemente solo se podrá llegar a través de la realización de estudios multicéntricos, prospectivos y randomizados- intentar aportar algún dato sobre aquellos factores que potencialmente podrían predecir el resultado de la cirugía puede ser interesante. En nuestro Servicio utilizamos la discectomía y artrodesis intersomática por vía anterior, mediante técnica de Cloward, para el tratamiento de los pacientes que presentan patología discal o espondilótica del raquis cervical, con un compromiso fundamentalmente anterior de las estructuras mielorradiculares. En el presente trabajo realizamos un análisis retrospectivo multivariante de los pacientes afectos de mielopatía cervical intervenidos por esta vía, con especial hincapié en los factores que condicionan el resultado clínico a largo plazo.

\section{Pacientes y métodos}

A partir del registro informatizado del Servicio de Admisión y Documentación Clínica del Hospital Universitario "Marqués de Valdecilla" se obtuvieron los pacientes intervenidos por primera vez mediante descompresión anterior y artrodesis intersomática, según técnica de Cloward, en el periodo de tiempo comprendido entre enero de 1984 y diciembre de 1997, con un seguimiento postoperatorio superior a un año. Como resultado se revisaron las historias clínicas de un total de 206 pacientes, de los cuales 54 habían sido operados como consecuencia de un cuadro clínico de mielopatía cervical, siendo uno de ellos excluido por tratarse de un caso de presentación aguda y origen traumático. Se realiza un análisis retrospectivo de las características epidemiológicas y clínicas, complicaciones acaecidas y resultado de la cirugía de los 53 pacientes restantes.

\section{Evaluación clínica preoperatoria}

Se describen los hallazgos clínicos y se agrupa a los pacientes siguiendo la escala de $\operatorname{Nurick}^{53}$ (tabla 1) y la escala de la Japanese Orthopaedic Association ${ }^{29}$, en su forma modificada para poblaciones occidentales (mJOA) introducida por Benzel y cols (tabla 2) . $^{4}$ Ambas se han demostrado útiles para la evaluación de la mielopatía cervical espondilótica, la primera por su sencillez y la segunda por aportar una valoración semicuantitativa y presentar una alta fiabilidad intra e interobservador ${ }^{74}$.

\section{Técnica quirúrgica y cuidados postoperatorios}

Todos los pacientes fueron intervenidos con idéntica técnica quirúrgica y recibieron el mismo tipo de cuidados postoperatorios, según hemos descrito en una publicación previa $^{41}$.

\section{Resultado clínico}

El resultado clínico es evaluado utilizando las escalas de Nurick y de la JOA en el momento de la última exploración en consultas externas reflejada en la historia clínica. Se define la razón de recuperación según la fórmula descrita por Hirabayashi a partir de las puntuaciones pre y postoperatoria (tabla 3$)^{29}$. El resultado de la cirugía se categoriza como bueno si se ha producido un incremento en el grado funcional en la escala de Nurick y como malo si el paciente sigue igual o ha empeorado.

\section{Resultado radiológico}

Se revisaron las radiografías cervicales obtenidas al 
Tabla 2

Escala Modificada de la Asociación de Ortopedia Japonesa (mJOA)

Puntuación

FUNCIÓN MOTRIZ DE EESS (Extremidades superiores)

Incapaz de alimentarse solo

0

Incapaz de usar cuchillo y tenedor, pero capaz de usar la cuchara

Usa los cubiertos con mucha dificultad

2

Usa los cubiertos con poca dificultad

3

Sin alteraciones

FUNCIÓN MOTRIZ DE EEII (Extremidades inferiores)

Incapaz de caminar

0

Necesita ayuda para caminar en suelo plano

1

Necesita utilizar el pasamanos al subir o bajar escalaras

Inestabilidad

2

Sin alteraciones

\section{DÉFICIT SENSITIVO}

\section{Extremidad superior}

Grave / Dolor

Leve

Sin déficit

Tronco

Grave / Dolor

Leve

Sin déficit

Extremidad inferior

Grave / Dolor

Leve

Sin déficit

\section{FUNCIÓN VESICAL}

Nula

0

Dificultad miccional grave (retención ocasional)

Dificultad miccional leve (poliacuria, urgencia urinaria)

Sin alteraciones

TOTAL

Tabla 3

Razón de recuperación de Hirabayashi

$$
\text { Razón de recuperación }=\frac{\text { Puntuación final }- \text { Puntuación inicial }}{17-\text { Puntuación inicial }} \times 100
$$


Tabla 4

Antecedentes personales

Traumatismo cervical

Dolor cervical crónico

Factores de riesgo vascular

Síndrome ansioso-depresivo

Fijación posterior C1-C2

Discectomía simple

año de la cirugía, en posición neutra, flexo-extensión e inclinación lateral del cuello, determinando la correcta fusión (ausencia de movilidad en el segmento intervenido en todas las proyecciones radiológicas) y la presencia de cifosis postquirúrgica (angulación superior a $\operatorname{los} 5^{\circ}$ en el segmento intervenido).

\section{Análisis estadístico}

Para el análisis estadístico empleamos el programa SPSS para Windows en su versión 8.0 (SPSS Inc, 1997), utilizando los diferentes test estadísticos según se encontrasen indicados (análisis multivariante y análisis factorial mediante modelo GLM, regresión lineal, t de Student para igualdad de la media en muestras independientes y diferentes estadísticos no paramétricos).

\section{Resultados}

\section{Características epidemiológicas}

Se intervinieron 35 varones (66\%) y 18 mujeres $(34 \%)$ con una relación varón:mujer de 1.94:1. El paciente más joven tenía 29 años y el de mayor edad 77 años, con una
$\mathrm{n}=9$
$17.0 \%$
$\mathrm{n}=7$
$13.2 \%$
$\mathrm{n}=5$
$9.4 \%$
$\mathrm{n}=2$
$3.8 \%$
$\mathrm{n}=1$
$1.9 \%$
$\mathrm{n}=1$
$1.9 \%$

media de 50.4 años, una mediana de 50 años y una desviación estándar de 12.8 años.

\section{Antecedentes Personales}

Los antecedentes personales de interés de los pacientes intervenidos se muestran en la tabla 4.

\section{Tiempo de Evolución}

El periodo sintomático previo a la consulta neuroquirúrgica fue variable, con una media de 16.70 meses desde el inicio de la clínica y una desviación estándar de 26.85 meses. Aunque, tal y como muestra la figura 1, prácticamente la mitad de los pacientes tenía una evolución inferior a los 6 meses, como contrapunto debemos señalar que casi una cuarta parte de ellos había presentado clínica por un periodo igual o superior a los 2 años antes de ser remitido al especialista.

\section{Clínica}

El $75.5 \%$ de los enfermos se presentaron como una mielopatía pura, mientras que en el $24.5 \%$ restante se identificaban, además, síntomas o signos de tipo radicular

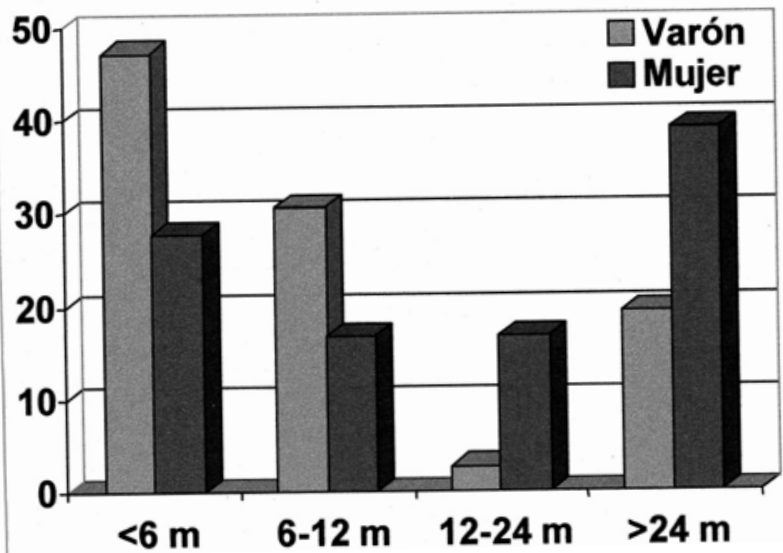

\begin{tabular}{|c|c|c|c|}
\cline { 2 - 4 } \multicolumn{1}{c|}{} & Total & Varones & Mujeres \\
\hline \hline < 6 meses & $\begin{array}{c}39.6 \% \\
(\mathrm{n}=21)\end{array}$ & $\begin{array}{c}45.7 \% \\
(\mathrm{n}=16)\end{array}$ & $\begin{array}{c}27.8 \% \\
(\mathrm{n}=5)\end{array}$ \\
\hline \multirow{2}{*}{ 6-12 meses } & $26.4 \%$ & $31.4 \%$ & $16.7 \%$ \\
& $(\mathrm{n}=14)$ & $(\mathrm{n}=11)$ & $(\mathrm{n}=3)$ \\
\hline 1-2 años & $7.5 \%$ & $2.9 \%$ & $16.7 \%$ \\
$(\mathrm{n}=4)$ & $(\mathrm{n}=1)$ & $(\mathrm{n}=3)$ \\
\hline > 2 años & $26.4 \%$ & $20.0 \%$ & $38.9 \%$ \\
$(\mathrm{n}=14)$ & $(\mathrm{n}=7)$ & $(\mathrm{n}=7)$ \\
\hline
\end{tabular}

Figura 1. Duración de la clínica en los pacientes intervenidos. Obsérvese que casi el $40 \%$ de las mujeres presentaban clínica por un periodo superior a los 24 meses, siendo el sexo un factor relacionado estadísticamente con el tiempo de evolución de la enfermedad $(p=0.030)$. 
Tabla 5

Características clínicas

\begin{tabular}{|c|c|c|}
\hline \multicolumn{3}{|c|}{ Déficit motor } \\
\hline No déficit motor & $\mathrm{n}=15$ & $28.3 \%$ \\
\hline Paraparesia & $\mathrm{n}=26$ & $49.1 \%$ \\
\hline Tetraparesia & $\mathrm{n}=8$ & $15.1 \%$ \\
\hline Hemiparesia & $\mathrm{n}=4$ & $7.5 \%$ \\
\hline \multicolumn{3}{|c|}{ Déficit sensitivo } \\
\hline No déficit sensitivo & $\mathrm{n}=21$ & $39.6 \%$ \\
\hline Hipopalestesia sublesional & $\mathrm{n}=15$ & $28.3 \%$ \\
\hline Nivel sensitivo completo sublesional & $\mathrm{n}=11$ & $20.8 \%$ \\
\hline Nivel sensitivo suspendido & $\mathrm{n}=1$ & $1.9 \%$ \\
\hline Hemihipoestesia sublesional & $\mathrm{n}=5$ & $9.4 \%$ \\
\hline \multicolumn{3}{|c|}{ Alteración del control de esfínteres } \\
\hline No alteración esfinteríana & $\mathrm{n}=42$ & $79.2 \%$ \\
\hline Urgencia urinaria & $\mathrm{n}=8$ & $15.1 \%$ \\
\hline Retención urinaria ocasional & $\mathrm{n}=2$ & $3.8 \%$ \\
\hline Retención urinaria (sonda vesical) & $\mathrm{n}=1$ & $1.9 \%$ \\
\hline \multicolumn{3}{|c|}{ Signos piramidales } \\
\hline Alteración de los ROTs & $\mathrm{n}=49$ & $92.4 \%$ \\
\hline Hiperreflexia & $n=29$ & $54.7 \%$ \\
\hline Clonus & $n=20$ & $37.7 \%$ \\
\hline Hipertonía & $\mathrm{n}=51$ & $96.3 \%$ \\
\hline Babinski & $\mathrm{n}=31$ & $58.5 \%$ \\
\hline Hoffman / Tröemner & $\mathrm{n}=22$ & $41.5 \%$ \\
\hline
\end{tabular}

(mielorradiculopatía), que orientaban hacia el origen cervical de la clínica. El motivo mayoritario de consulta fue el deterioro de la marcha, que aquejaba el $90.6 \%$ de los enfermos. Se objetivo déficit motor en el $72.2 \%$ de los pacientes, sensitivo en el $61.1 \%$ y alteración en el control de esfínteres en aproximadamente una cuarta parte. Prácticamente la totalidad de los enfermos presentaba signos piramidales en la exploración (tabla 5). El signo de Lhermitte únicamente se observó en el 3.8\% de los pacientes.

Si agrupamos los hallazgos clínicos, la forma de presentación más frecuente fue el síndrome cordonal anterior (62.3\%), seguido del síndrome centro-medular (22.6\%) y del síndrome de hemisección medular de Brown-Sequard $(15.1 \%)$.

La gravedad clínica se graduó utilizando la escala de Nurick y la escala modificada para poblaciones occidentales de la Japanese Orthopaedic Association (mJOA). Los resultados se muestran en la figura 2, presentando ambas escalas una correlación elevada, con una $r$ de Pearson=$0.864(\mathrm{p}<0.001)$.

\section{Neuroimagen}

Se realizó mielografía al 39.6\% de los pacientes $(n=21)$, tomografía computerizada al $26.4 \%(\mathrm{n}=14)$ y resonancia 


\begin{tabular}{|l|c|}
\hline \multicolumn{2}{|c|}{ Escala de Nurick } \\
\hline Grado 0 & - \\
\hline Grado 1 & $9.4 \%(\mathrm{n}=5)$ \\
\hline Grado 2 & $45.3 \%(\mathrm{n}=24)$ \\
\hline Grado 3 & $34.0 \%(\mathrm{n}=18)$ \\
\hline Grado 4 & $9.4 \%(\mathrm{n}=5)$ \\
\hline Grado 5 & $1.9 \%(\mathrm{n}=1)$ \\
\hline
\end{tabular}

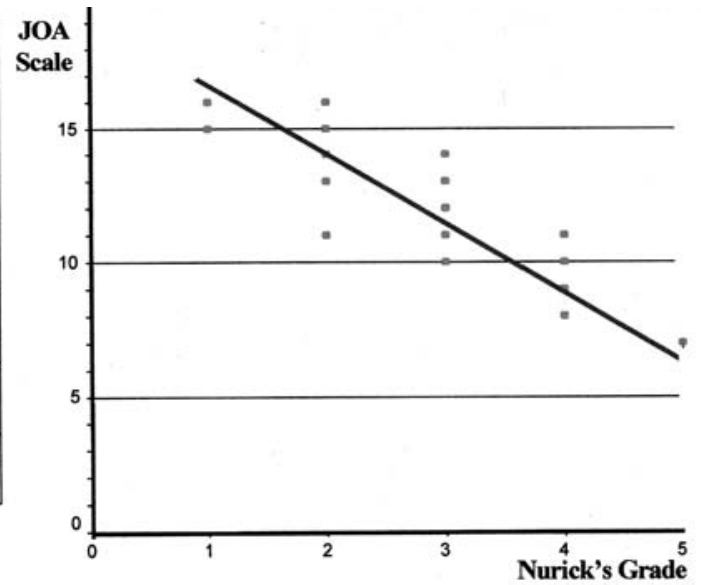

\begin{tabular}{|c|c|c|c|}
\hline \multicolumn{4}{|c|}{ Escala de la JAO } \\
\hline 0 & - & 9 & $3.8 \%(\mathrm{n}=2)$ \\
\hline 1 & - & 10 & $7.5 \%(\mathrm{n}=4)$ \\
\hline 2 & - & 11 & $15.1 \%(\mathrm{n}=8)$ \\
\hline 3 & - & 12 & $5.7 \%(\mathrm{n}=3)$ \\
\hline 4 & - & 13 & $15.1 \%(\mathrm{n}=8)$ \\
\hline 5 & - & 14 & $28.3 \%(\mathrm{n}=15)$ \\
\hline 6 & - & 15 & $11.3 \%(\mathrm{n}=6)$ \\
\hline 7 & $1.9 \%(\mathrm{n}=1)$ & 16 & $9.4 \%(\mathrm{n}=5)$ \\
\hline 8 & $1.9 \%(\mathrm{n}=1)$ & 17 & - \\
\hline
\end{tabular}

Figura 2. Estado neurológico prequirúrgico. La mayor parte de los pacientes presentaban una mielopatía leve-moderada. La correlación entre la escala de Nurick y la escala modificada de la asociación de ortopedia japonesa (JOA) es excelente ( $r$ de Pearson=-0.864).

magnética al $61.1 \%(n=33)$. En el $100 \%$ se realizaron, de forma complementaria, estudios radiográficos simples consistentes en proyecciones antero-posterior y lateral en posición neutra del cuello, y dinámicos en flexo-extensión. Tras la valoración conjunta de las pruebas neurorradiológicas se llegó al diagnóstico de hernia discal en 23 pacientes (44.4\%) y de espondilosis en 30 pacientes (55.6\%).

De los pacientes estudiados mediante RM el $69.70 \%$ $(n=23)$ presentaba hiperseñal intramedular en las secuencias potenciadas en T2. En otros dos pacientes (6.06\%) se objetivó la presencia de una cavidad siringomiélica, presentando uno de estos pacientes la clínica típica de nivel medular suspendido y disociación sensitiva.

\section{Estudio neurofisiológico}

Se le realizó electromiograma (EMG), conducción nerviosa (ENG), potenciales evocados somatosensoriales (PESS) y estimulación magnética cortical transcraneal (EMC) a 21 pacientes $(38.89 \%)$. Tal y como muestra la tabla 6 , el $71.43 \%$ de los pacientes $(\mathrm{n}=15)$ presentaban alteración de la conducción medular, mientras que en un $19.05 \%(n=4)$ el estudio era estrictamente normal.

\section{Cirugía}

38 pacientes $(71.7 \%)$ fueron operados de un único espacio. El espacio intervenido con mayor frecuencia fue el C5-C6 (39.6\%) seguido del C6-C7 (17.0\%). En 13 pacientes $(24.5 \%)$ la cirugía se realizó sobre dos espacios, correspondiendo el $13.2 \%$ a la artrodesis de los espacios C4-C5 y C5-C6, y en 2 pacientes (3.8\%) sobre tres espacios (figura 3). Con respecto a la altura de los niveles intervenidos, agrupamos los espacios en segmento cervical alto (C3-C4 y C4-C5) y segmento cervical bajo (C5-C6, C6-C7 y $\mathrm{C} 7-\mathrm{T} 1)$.

El control radiológico, realizado a las 24 horas de la cirugía, demostró la correcta colocación del injerto óseo en el $92.5 \%$ de los pacientes $(\mathrm{n}=49)$. En 3 pacientes $(5.7 \%)$ existía desalineación anterior y en 1 paciente $(1.9 \%)$ desalineación posterior, que no superaba los $2 \mathrm{~mm}$. El análisis multivariante no detectó ninguna variable que se correlacionara de forma significativa con la incorrecta colocación del injerto.

\section{Complicaciones (tabla 7)}

18 pacientes (34.0\%) presentaron algún tipo de complicación. El análisis multivariante correlacionó significativamente el desarrollo de complicaciones con la presencia de factores de riesgo vascular $(\mathrm{p}=0.001)$, encontrándose el segmento cervical intervenido al límite de la significación estadística $(\mathrm{p}=0.056)$. El análisis univariante mediante test exacto de Fisher confirmó que los pacientes

Tabla 6

Estudio neurofisiológico

Alteración de la conducción medular (PESS o EMC)

$\mathrm{n}=15$

$71.43 \%$

Patrón inespecífico

$\mathrm{n}=1$

$4.76 \%$

Radiculopatía

$\mathrm{n}=1$

$4.76 \%$

Normal $\mathrm{n}=4$

$19.05 \%$ 


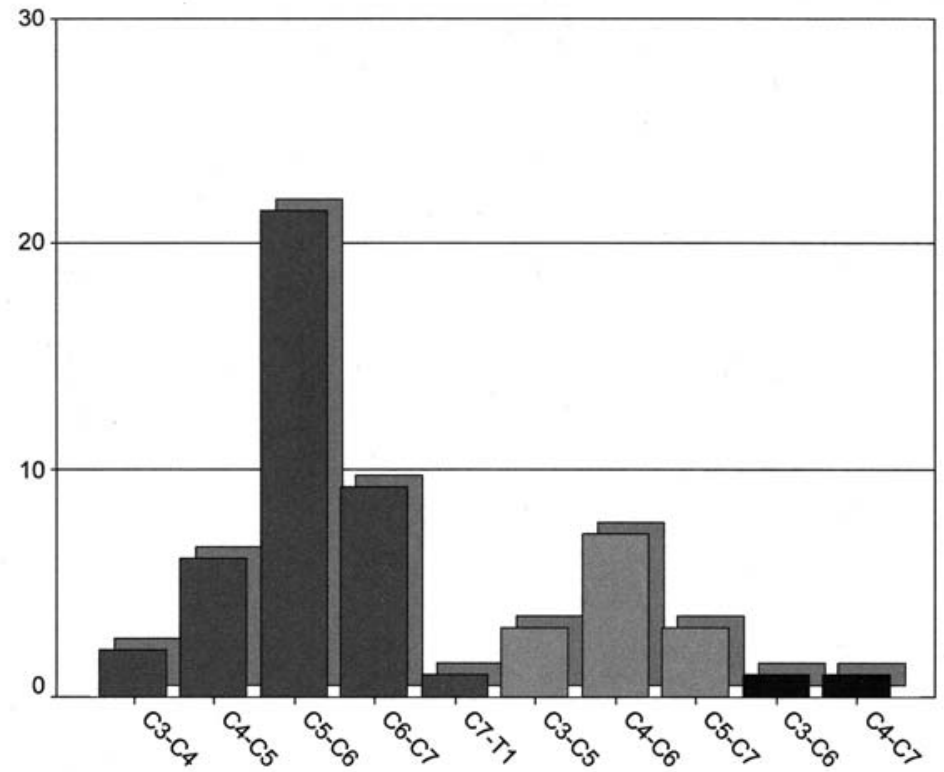

\begin{tabular}{|l|r|c|}
\cline { 2 - 3 } \multicolumn{1}{c|}{} & \multicolumn{1}{c|}{$\mathbf{~}$} & Porcentaje \\
\hline 1 espacio & $\mathbf{3 8}$ & $\mathbf{7 1 . 7 \%}$ \\
\hline C3-C4 & 2 & $3.8 \%$ \\
\hline C4-C5 & 5 & $9.4 \%$ \\
\hline C5-C6 & 21 & $39.6 \%$ \\
\hline C6-C7 & 9 & $17.0 \%$ \\
\hline C7-T1 & 1 & $1.9 \%$ \\
\hline $\mathbf{2}$ espacios & $\mathbf{1 3}$ & $\mathbf{2 4 . 5 \%}$ \\
\hline C3-C4 + C4-C5 & 3 & $5.7 \%$ \\
\hline C4-C5 + C5-C6 & 7 & $13.2 \%$ \\
\hline C5-C6 + C6-C7 & 3 & $5.7 \%$ \\
\hline 3 espacios & $\mathbf{2}$ & $\mathbf{3 . 8} \%$ \\
\hline C3-C4 + C4-C5 + C5-C6 & 1 & $1.9 \%$ \\
\hline C4-C5 + C5-C6 + C6-C7 & 1 & $1.9 \%$ \\
\hline TOTAL & $\mathbf{5 3}$ & $\mathbf{1 0 0 . 0} \%$ \\
\hline
\end{tabular}

Figura 3. Distribución de los espacios intervenidos.

con factores de riesgo vascular presentaban algún tipo de complicación con mayor frecuencia que los pacientes sin factores de riesgo vascular $(100 \%$ vs. $27.1 \%, p=0.003)$. Igualmente el test $\chi^{2}$ de Pearson mostró que los pacientes intervenidos del segmento cervical superior se complicaban más que los intervenidos del segmento cervical inferior ( $58.3 \%$ vs. $25.7 \%, \mathrm{p}=0.04)$. La figura 4 ilustra gráficamente los resultados.

\section{A) Complicaciones intraoperatorias}

En 2 pacientes $(3.8 \%)$ se produjeron complicaciones intraoperatorias incidentales, consistentes en una apertura dural $(1.9 \%)$ y una apertura esofágica $(1.9 \%)$. Curiosamente ninguno de estos pacientes presentó complicaciones adicionales transitorias o definitivas, y el incidente quirúrgico se resolvió sin secuelas. La apertura dural se selló con adhesivo de fibrina (Tissucol®) y la apertura esofágica mediante sutura por planos con material reabsorbible.

Tabla 7

\section{Complicaciones}

\begin{tabular}{|c|c|c|c|c|}
\hline & $\mathbf{N}$ & Transitorias & Definitivas & Porcentaje \\
\hline Neurológicas & 10 & $5(9.4 \%)$ & $5(9.4 \%)$ & $18.9 \%$ \\
\hline Mielopatía & 6 & $2(3.8 \%)$ & $4(7.5 \%)$ & $11.3 \%$ \\
\hline Radiculopatía & 2 & $1(1.9 \%)$ & $1(1.9 \%)$ & $3.8 \%$ \\
\hline Parálisis del N. recurrente & 1 & $1(1.9 \%)$ & - & $1.9 \%$ \\
\hline Síndrome de Horner & 1 & $1(1.9 \%)$ & - & $1.9 \%$ \\
\hline Transitorias no neurológicas & 7 & & & $13.2 \%$ \\
\hline Disfagia & 5 & \multirow{3}{*}{\multicolumn{2}{|c|}{-}} & $9.4 \%$ \\
\hline Infección respiratoria & 1 & & & $1.9 \%$ \\
\hline Infección urinaria & 1 & & & $1.9 \%$ \\
\hline Intraoperatorias & 2 & & & $3.8 \%$ \\
\hline Duramadre & 1 & \multirow{2}{*}{\multicolumn{2}{|c|}{ - }} & $1.9 \%$ \\
\hline Esófago & 1 & & & $1.9 \%$ \\
\hline
\end{tabular}




\begin{tabular}{|c|c|c|c|c|c|}
\hline & & \multicolumn{2}{|c|}{ Complicaciones } & \multicolumn{2}{|c|}{ Análisis estadístico } \\
\hline & & $S i$ & No & Multivariante & Univariante \\
\hline \multirow{2}{*}{$\begin{array}{c}\text { Factores riesgo } \\
\text { vascular }\end{array}$} & No & $27.1 \%$ & $72.9 \%$ & \multirow{2}{*}{$p=0.001$} & \multirow{2}{*}{$p=0.003$} \\
\hline & $S i$ & $100 \%$ & - & & \\
\hline \multirow{2}{*}{ Segmento cervical } & Sup & $58.3 \%$ & $41.7 \%$ & \multirow{2}{*}{$p=0.056$} & \multirow{2}{*}{$p=0.04$} \\
\hline & $\operatorname{Inf}$ & $25.7 \%$ & $74.3 \%$ & & \\
\hline
\end{tabular}

\section{Complicaciones}

(\%)

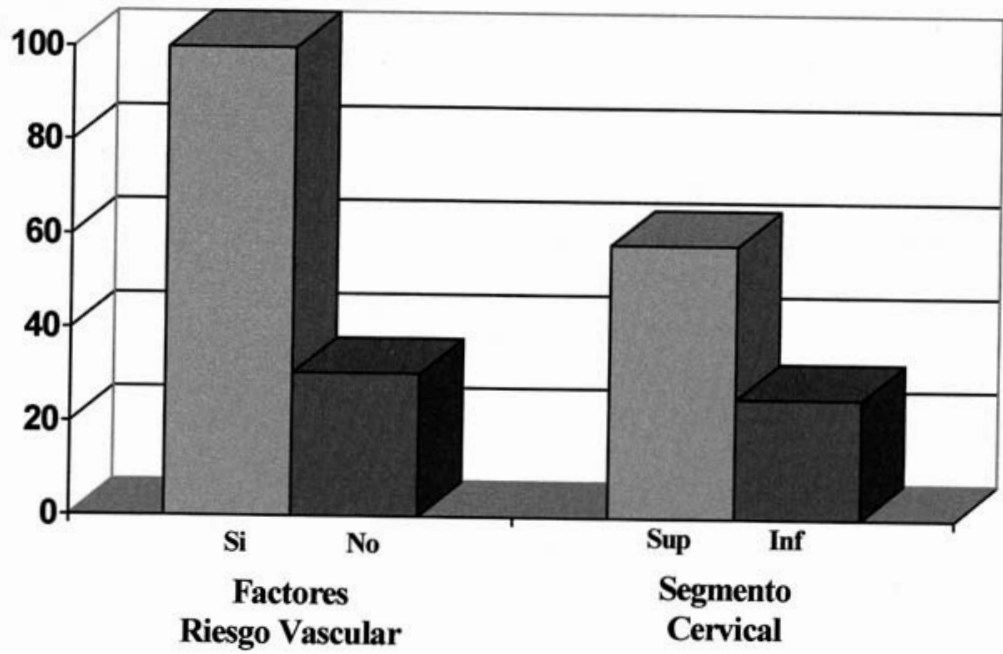

Figura 4. Factores relacionados con el desarrollo de complicaciones.

\section{B) Complicaciones transitorias no neurológicas}

La complicación transitoria más frecuente fue la disfagia postoperatoria, que se presentó en el $9.4 \%$ de los pacientes $(n=5)$. En el análisis multivariante, el desarrollo de disfagia postoperatoria se correlacionó únicamente con el segmento cervical intervenido $(\mathrm{p}=0.002)$. El test $\chi^{2}$ de Pearson confirmó que los pacientes intervenidos del segmento cervical superior desarrollaban disfagia postoperatoria más frecuentemente que los intervenidos del segmento inferior $(36.4 \%$ vs. $2.9 \%$. $p=0.009)$, con una odds ratio de 12.73 en la estimación de riesgo (intervalo de confianza del $95 \%=1.58-102.28)$.

2 pacientes $(3.8 \%)$ presentaron complicaciones infecciosas, todas ellas sistémicas: 1 infección respiratoria $(1.9 \%)$, y una infección urinaria $(1.9 \%)$.

\section{C) Complicaciones neurológicas}

En el $18.9 \%$ de los pacientes se objetivó deterioro neurológico tras la cirugía, siendo éste definitivo en el 9.4\%. Al analizar globalmente las complicaciones neurológicas, el modelo multivariante detectó únicamente, como variable significativa, la presencia de factores de riesgo vascular $(\mathrm{p}<0.001)$ El análisis univariante mediante el test exacto de Fisher confirmó que los pacientes con factores de riesgo vascular se deterioraban con mayor frecuencia $(80.0 \%$ vs. $12.5 \%, \mathrm{p}=0.003)$. La estimación de riesgo arrojó una odds ratio=28.00 (intervalo de confianza del $95 \%=2.66-294.28)$.

Sin embargo, al analizar por separado las complicaciones neurológicas transitorias y definitivas, se pone de manifiesto que los factores que influyen en ambas son diferentes. El análisis multivariante correlacionó significativamente el tiempo de evolución de los síntomas con la aparición de deterioro neurológico transitorio $(\mathrm{p}=0.037)$ y el cirujano que realizó la intervención $(\mathrm{p}=0.045)$. Tras someter estos

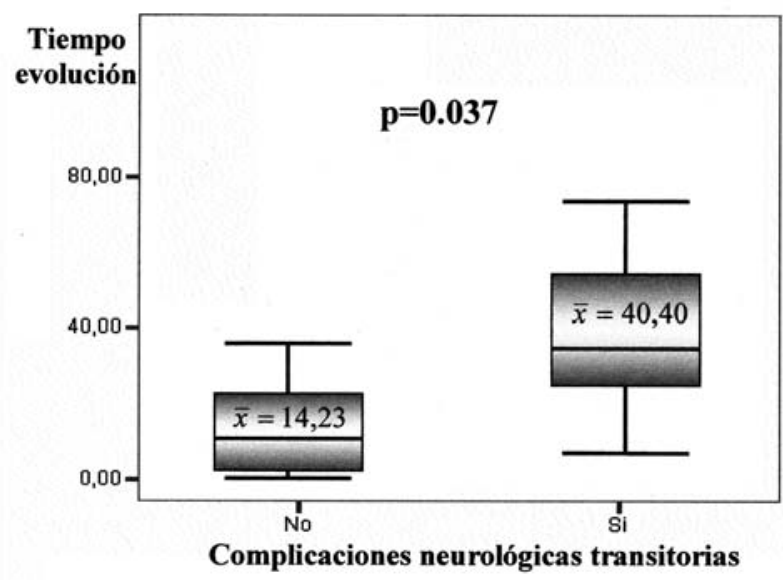

Figura 5. La duración prolongada de la clínica se relacionó con una mayor incidencia de complicaciones neurológicas transitorias. 


\begin{tabular}{|c|c|c|c|c|c|c|c|}
\cline { 2 - 8 } \multicolumn{1}{c|}{} & Cirujano 1 & Cirujano 2 & Cirujano 3 & Cirujano 4 & Cirujano 5 & Cirujano 6 & Cirujano 7 \\
\hline $\begin{array}{c}\text { Pacientes } \\
\text { intervenidos }\end{array}$ & 1 & 5 & 9 & 21 & 9 & 6 & 2 \\
\hline $\begin{array}{c}\text { Complicaciones } \\
\text { transitorias }\end{array}$ & $0 \%$ & $0 \%$ & $0 \%$ & $9.5 \%$ & $0 \%$ & $50 \%$ & $0 \%$ \\
\hline $\begin{array}{c}\text { Complicaciones } \\
\text { definitivas }\end{array}$ & $0 \%$ & $0 \%$ & $11.1 \%$ & $9.5 \%$ & $22.2 \%$ & $0 \%$ & $0 \%$ \\
\hline
\end{tabular}

\section{Complicaciones}

(\%)

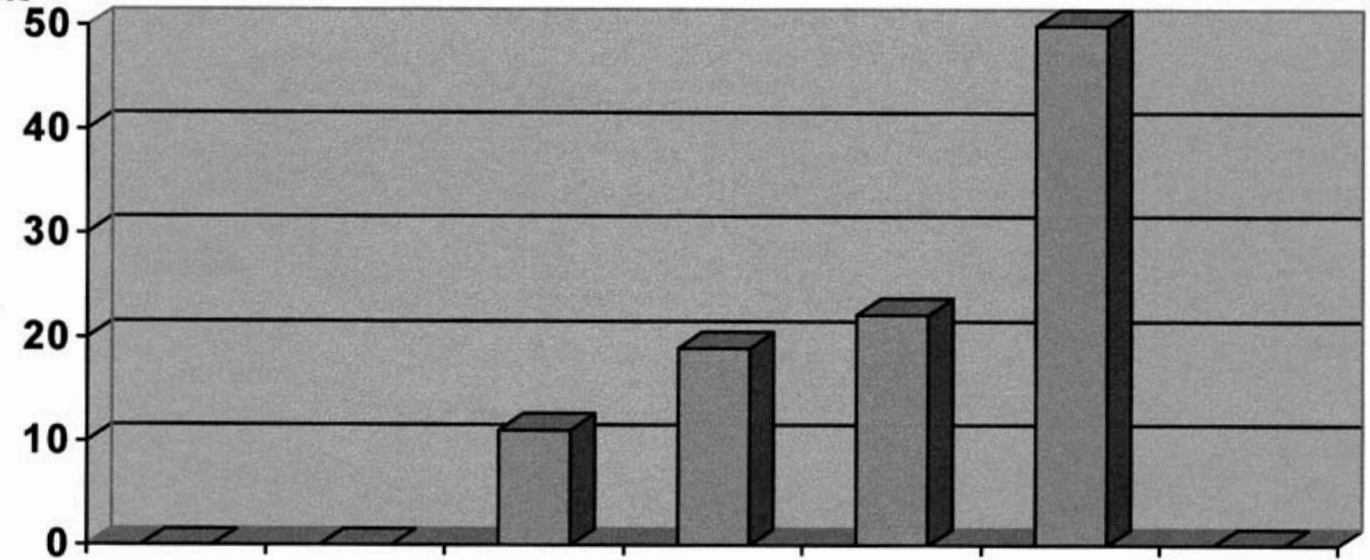

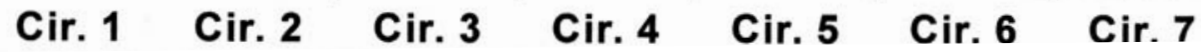

Figura 6. Distribución de las complicaciones de la cirugía según el cirujano que realizó la intervención.

factores al análisis univariante únicamente el primero resulto significativo. El tiempo medio de evolución de los síntomas en los pacientes que presentaron una complicación neurológica transitoria era mayor que en aquéllos que no la presentaron ( 40.40 meses vs. 14.23 meses) con una $\mathrm{p}=0.037$ en la prueba $\mathrm{t}$ de Student (figura 5). Respecto al cirujano responsable, los resultados se muestran gráficamente en la figura 6.
El modelo multivariante correlacionó significativamente el desarrollo de complicaciones neurológicas definitivas con la presencia de factores de riesgo vascular $(\mathrm{p}<0.001)$, encontrándose al borde de la significación estadística el síndrome clínico $(\mathrm{p}=0.096)$. El análisis univariante confirmó únicamente el primer factor (tabla 8). El 60\% de los pacientes con factores de riesgo vascular se deterioró neurológicamente de forma permanente tras la cirugía,

Tabla 8

Deterioro neurológico definitivo

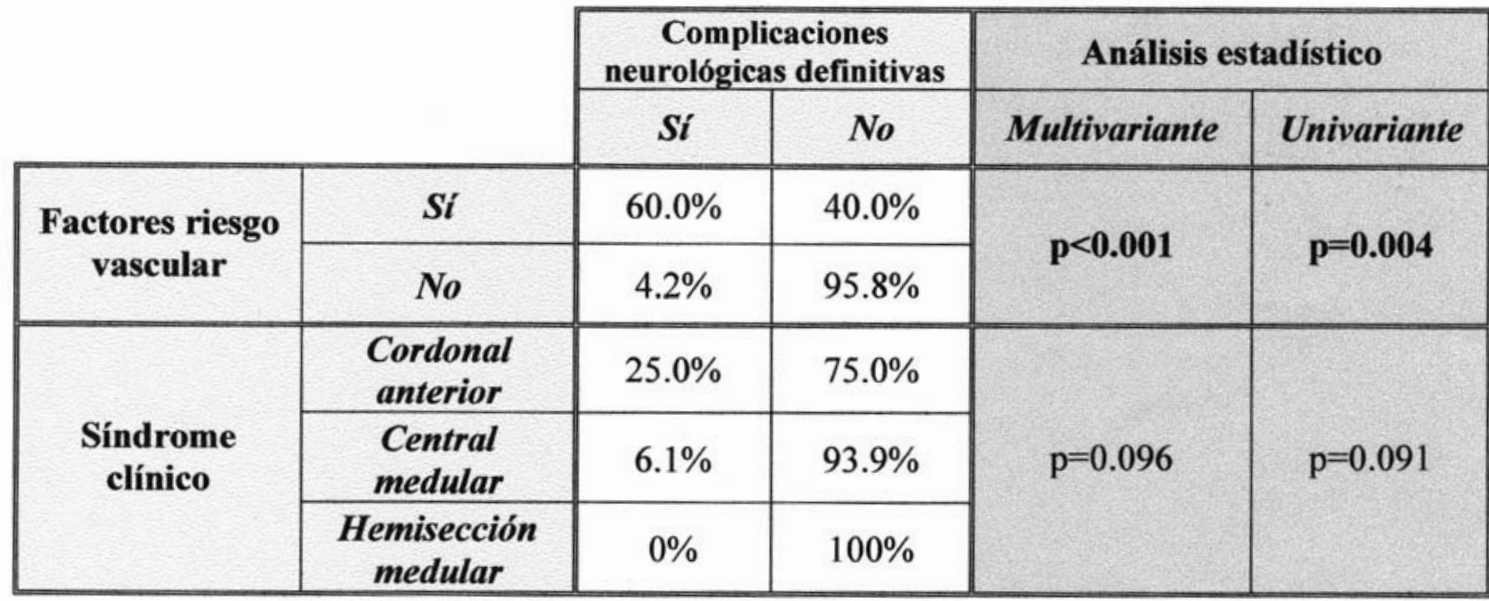


Tabla 9

Resultado radiológico

\begin{tabular}{|c|c|c|}
\cline { 2 - 3 } \multicolumn{1}{c|}{} & \multicolumn{1}{c|}{ P } & Porcentaje \\
\hline Fusión incorrecta & 4 & $\mathbf{7 . 4 \%}$ \\
\hline Pseudoartrosis & 3 & $5.6 \%$ \\
\hline Claudicación injerto & 1 & $1.9 \%$ \\
\hline Cifosis & 5 & $9.3 \%$ \\
\hline
\end{tabular}

frente al $4.2 \%$ de los pacientes sin factores de riesgo vascular (prueba exacta de Fisher, $\mathrm{p}=0.004$ ). La estimación de riesgo arrojó una odds ratio de 34.50 (intervalo de confianza del $95 \%=3.52-337.82$ ). Igualmente los pacientes que debutaron como síndrome centromedular mostraron mayor tendencia a deteriorase que aquéllos que se presentaron como síndrome cordonal anterior o hemisección medular (25.0\% vs. $6.1 \%$ vs. $0 \%)$, sin llegar a la significación estadística con una $\mathrm{p}=0.091$ en el test $\chi^{2}$ de Pearson con corrección iterativa de Montecarlo.

De todas las complicaciones neurológicas posibles, la más catastrófica para el paciente es el deterioro neurológico postquirúrgico de la mielopatía. El análisis multivariante detectó como factor significativo la presencia de factores de riesgo vascular $(\mathrm{p}<0.001)$, confirmando el análisis univariante mediante el test exacto de Fisher que los pacientes que presentaban factores de riesgo vascular se deterioraban con mayor frecuencia que aquéllos que no los presentaban (60\% vs. 6.3\%, $\mathrm{p}=0.008)$. La estimación de riesgo arrojó una odds ratio de 22.50 (intervalo de confianza del 95\%= 2.65-190.68). La figura 7 ilustra gráficamente los resultados.

\section{Resultado radiológico (tabla 9)}

La valoración de la radiología de control obtenida al año de la cirugía dio como resultado una tasa de correcta fusión del 92.5\% $(\mathrm{n}=49)$. En 3 pacientes se objetivó movilidad en el segmento intervenido, arrojando un porcentaje de pseudoartrosis del 5.7\%. En un enfermo (1.9\%) el injerto claudicó, por lo que precisó ser reintervenido. 5 pacientes (9.4\%) desarrollaron cifosis postquirúrgica.

\section{Deterioro}

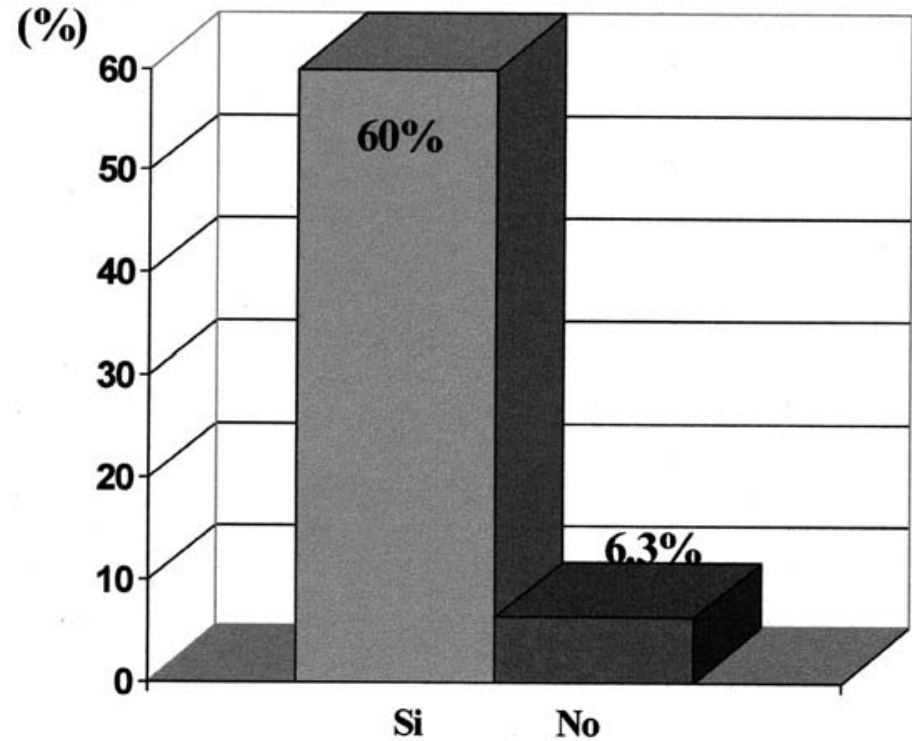

\begin{tabular}{|c|c|}
\hline $\begin{array}{c}\text { Análisis } \\
\text { multivariante }\end{array}$ & $\mathbf{p}<0.001$ \\
\hline $\begin{array}{c}\text { Análisis } \\
\text { univariante }\end{array}$ & $\mathbf{p}=\mathbf{0 . 0 0 8}$ \\
\hline $\begin{array}{c}\text { Odds } \\
\text { ratio }\end{array}$ & $\mathbf{2 2 . 5 0}$ \\
\hline
\end{tabular}

Factores riesgo vascular

Figura 7. Los factores de riesgo vascular se relacionaron con el deterioro de la mielopatía tras la cirugía. 


\begin{tabular}{|c|c|}
\hline \multicolumn{2}{|c|}{ Escala de Nurick } \\
\hline Grado 0 & $17.0 \%(\mathrm{n}=9)$ \\
\hline Grado 1 & $39.6 \%(\mathrm{n}=21)$ \\
\hline Grado 2 & $7.5 \%(\mathrm{n}=4)$ \\
\hline Grado 3 & $17.0 \%(\mathrm{n}=9)$ \\
\hline Grado 4 & $15.1 \%(\mathrm{n}=8)$ \\
\hline Grado 5 & $3.7 \%(\mathrm{n}=2)$ \\
\hline
\end{tabular}

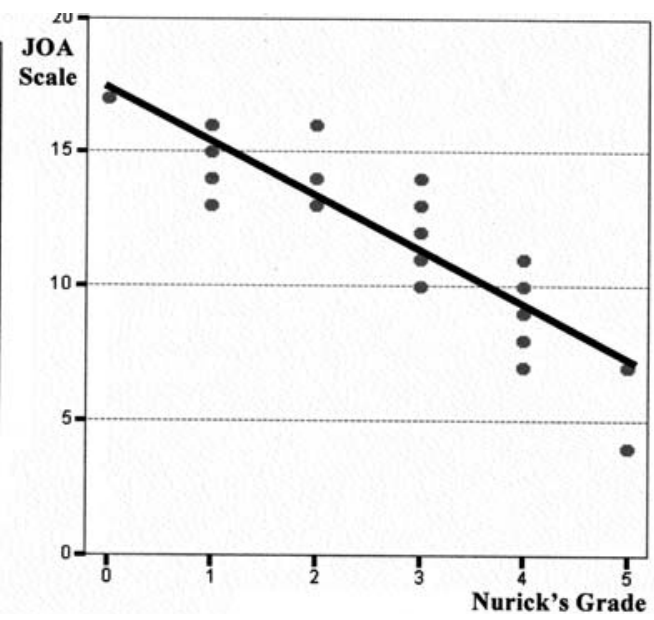

\begin{tabular}{|c|c|c|c|}
\hline \multicolumn{4}{|c|}{ Escala de la JAO } \\
\hline 0 & - & 9 & $7.5 \%(\mathrm{n}=4)$ \\
\hline 1 & - & 10 & $3.8 \%(\mathrm{n}=2)$ \\
\hline 2 & - & 11 & $5.7 \%(\mathrm{n}=3)$ \\
\hline 3 & - & 12 & $1.9 \%(\mathrm{n}=1)$ \\
\hline 4 & $1.9 \%(\mathrm{n}=1)$ & 13 & $11.3 \%(\mathrm{n}=6)$ \\
\hline 5 & - & 14 & $7.5 \%(\mathrm{n}=4)$ \\
\hline 6 & - & 15 & $15.1 \%(\mathrm{n}=8)$ \\
\hline 7 & $3.8 \%(\mathrm{n}=2)$ & 16 & $22.6 \%(\mathrm{n}=12)$ \\
\hline 8 & $1.9 \%(\mathrm{n}=1)$ & 17 & $17.0 \%(\mathrm{n}=9)$ \\
\hline \multicolumn{4}{|c}{} \\
\hline
\end{tabular}

Figura 8. Estado neurológico postquirúrgico. La correlación entre la escala de Nurik y la escala modificada de la asociación de ortopedia japonesa (JOA) es, de nuevo, excelente ( $r$ de Pearson=-0.938).

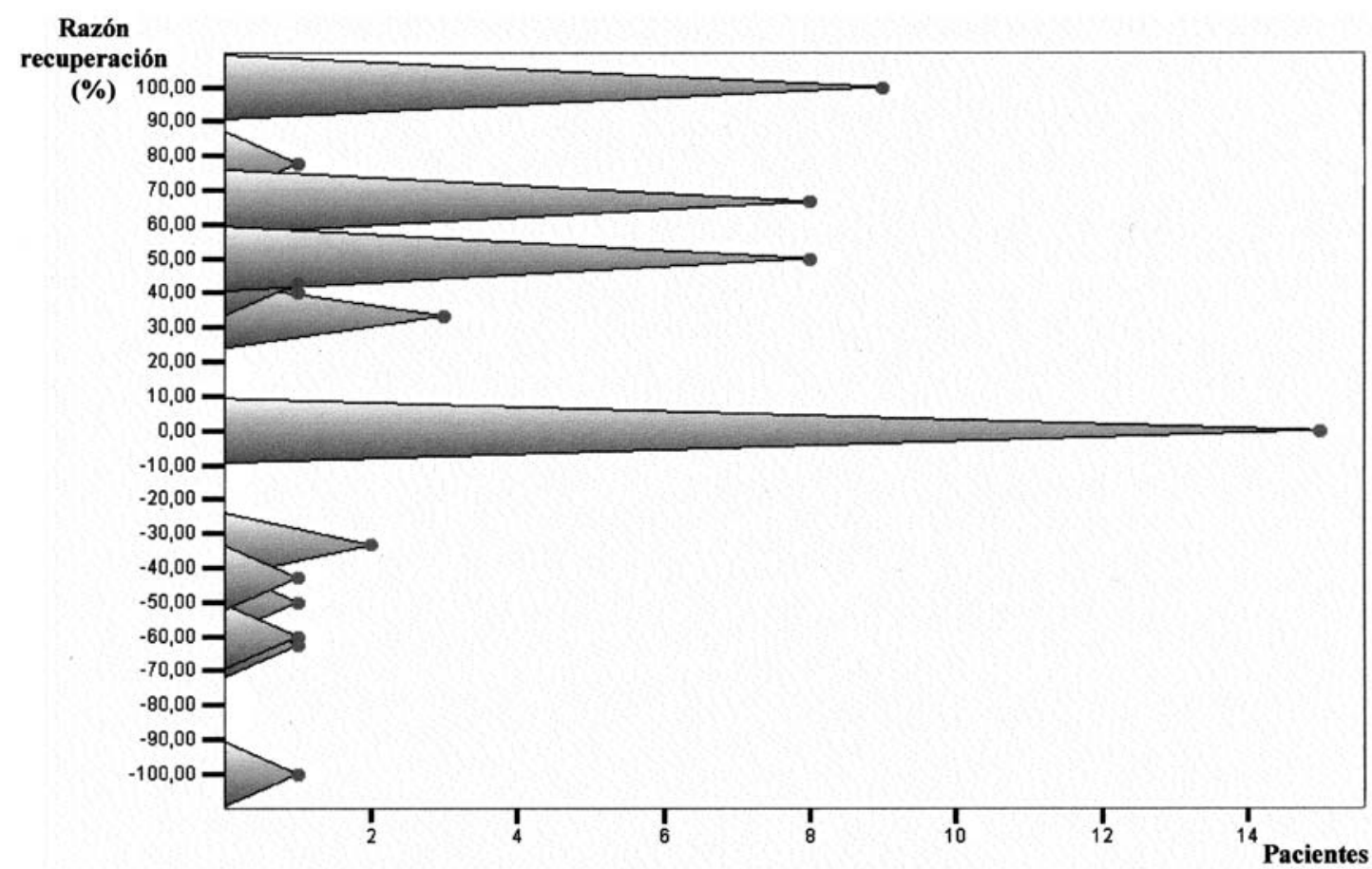

Figura 9. Distribución de los pacientes según la razón de recuperación de Hirabayashi.

\section{Resultado clínico}

El tiempo medio de seguimiento de los pacientes de esta serie es de 3 años (rango 2-14 años). En la figura 8 se ilustra el estado neurológico postquirúrgico en la última revisión de los pacientes en consultas externas. La correlación de la puntuación postoperatoria para la escala de Nurick y la escala de la JOA fue elevada, con una $\mathrm{r}$ de Pearson de $-0.938(\mathrm{p}<0.001)$. El resultado quirúrgico se categorizó como bueno en 34 de los 53 pacientes $(64.2 \%)$, mientras que el $35.8 \%$ restante $(n=19)$ no se benefició de la cirugía.
La puntuación media postoperatoria en la escala de la JOA fue $13.70,0.85$ puntos más que la preoperatoria (diferencia estadísticamente significativa con una $\mathrm{p}=0.005$, test $\mathrm{t}$ de Student para muestras apareadas). La media de la razón de recuperación de los pacientes intervenidos fue $32.30 \%$ (figura 9).

El análisis multivariante relacionó los siguientes factores con el resultado quirúrgico (figuras 10 y 11): edad ( $\mathrm{p}=0.008$ ), presencia de factores de riesgo vascular $(\mathrm{p}=0.031)$, tiempo de evolución ( $\mathrm{p}=0.002)$, estado neurológico prequirúrgico 


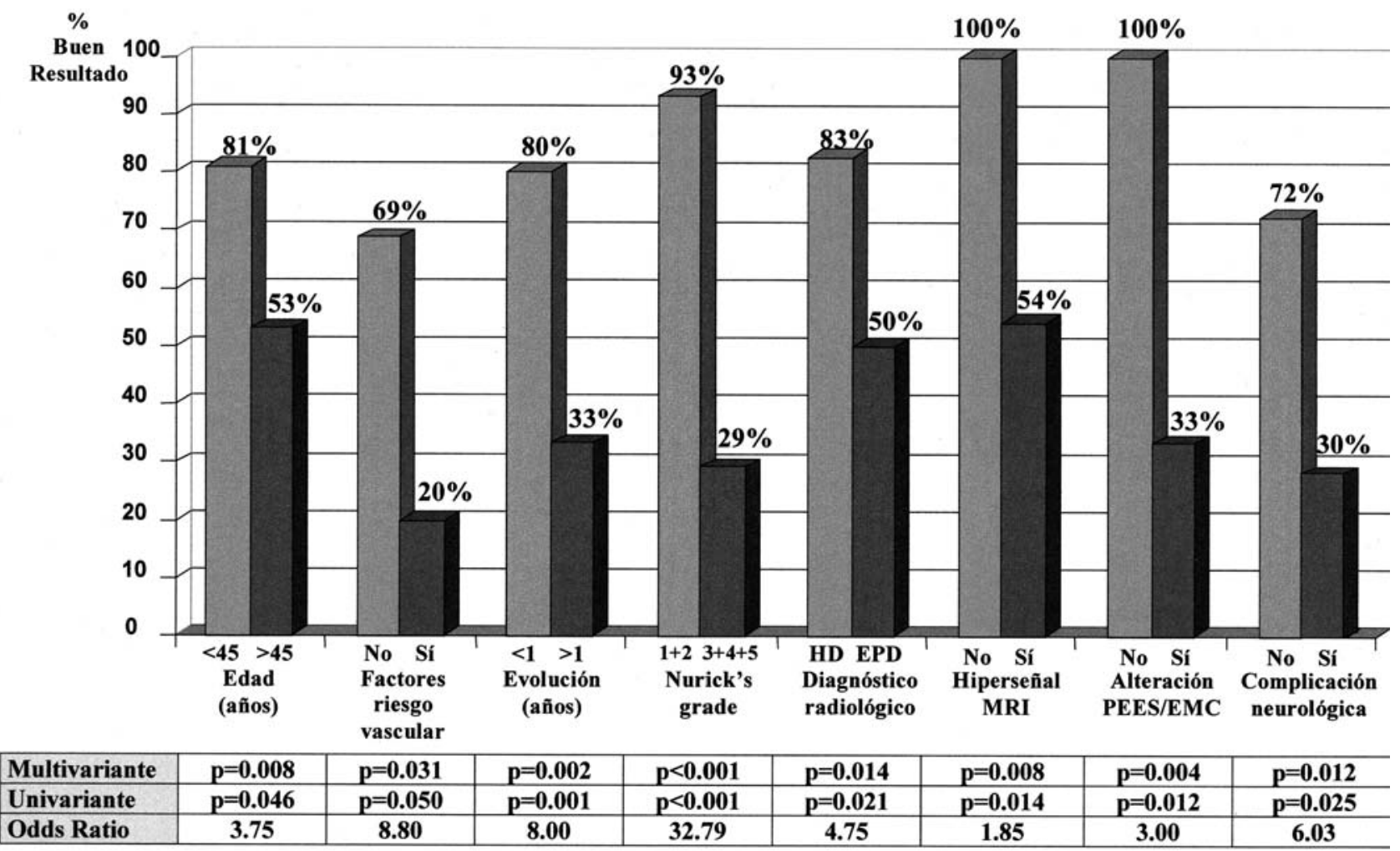

Figura 10. Factores relacionados con el resultado quirúrgico. $H D=$ Hernia discal. EPD=Espondilosis. $M R I=$ Resonancia magnética. PESS=Potenciales evocados somatosensoriales. EMC=Estimulación magnética transcraneal.

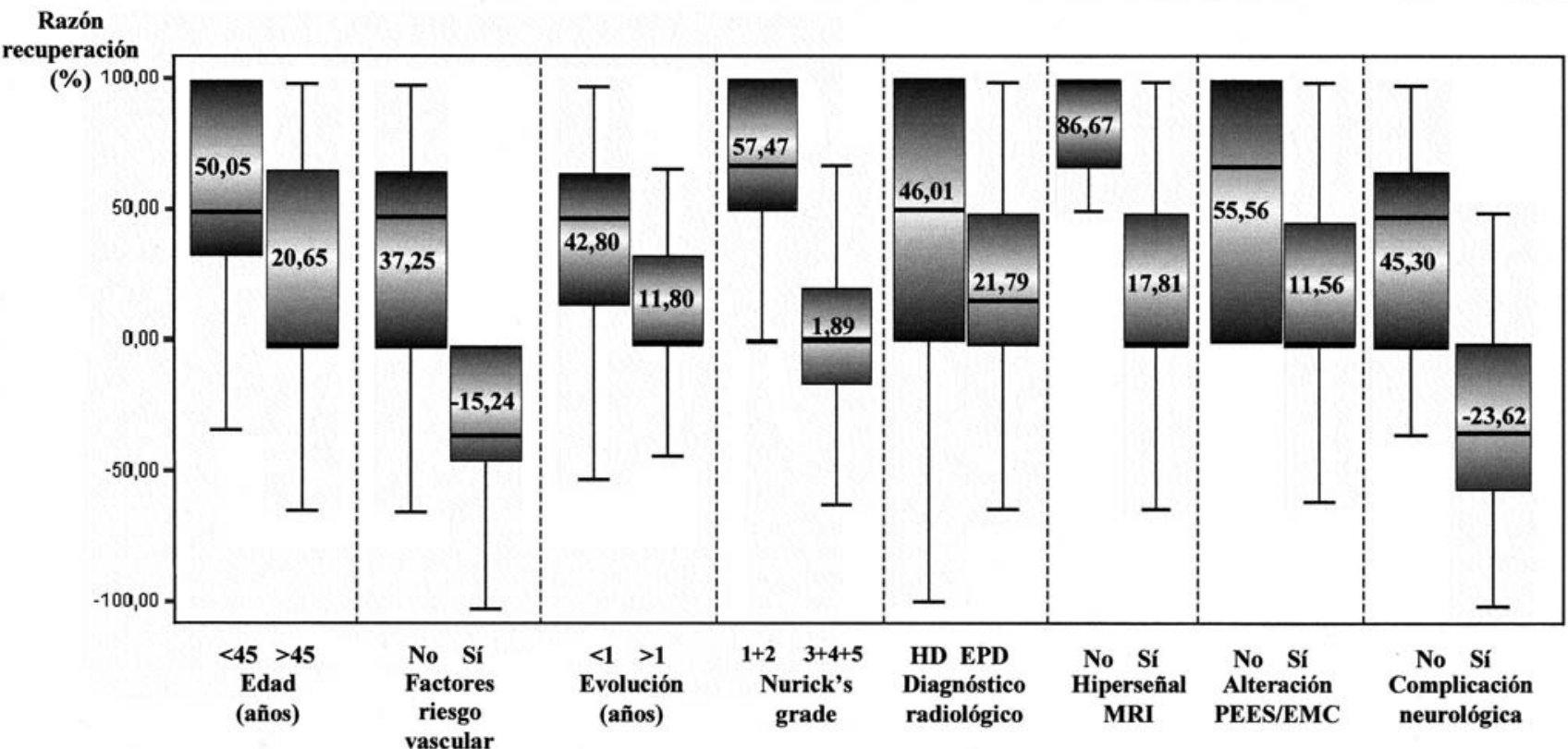

Figura 11. Factores relacionados con la razón de recuperación de Hirabayashi. HD=Hernia discal. EPD=Espondilosis. MRI=Resonancia magnética. PESS=Potenciales evocados somatosensoriales. EMC=Estimulación magnética transcraneal. 


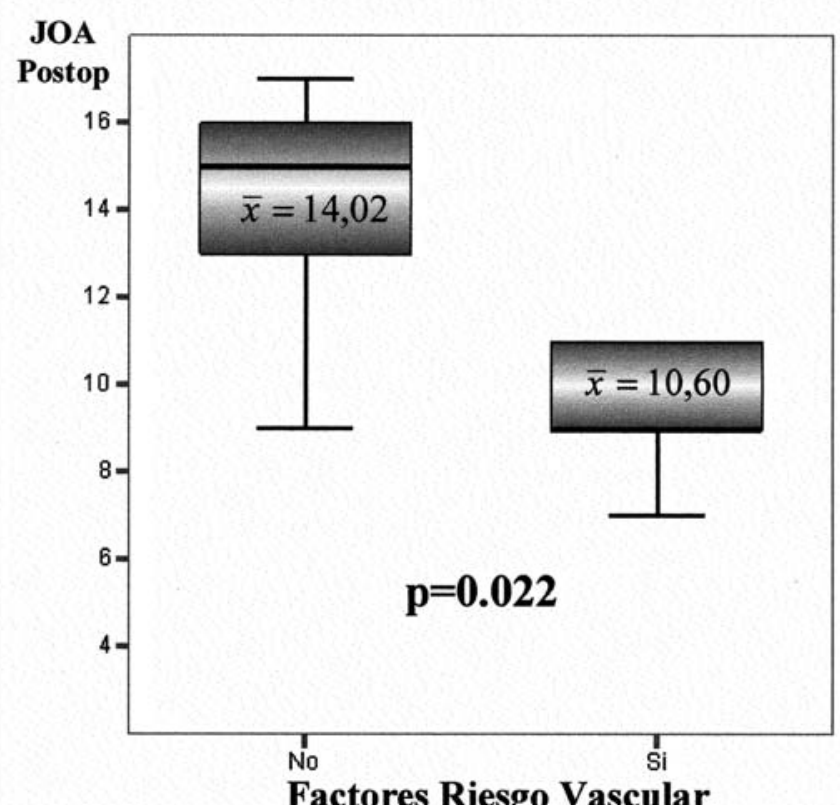

Factores Riesgo Vascular

Figura 12. Relación de los factores de riesgo vascular con el estado neurológico postquirúrgico según la escala de la asociación de ortopedia japonesa (JOA).

( $\mathrm{p}<0.001)$, diagnóstico neurorradiológico $(\mathrm{p}=0.014)$, presencia de hiperseñal medular en la resonancia magnética $(\mathrm{p}=0.008)$, alteración de la conducción medular en el estudio neurofisiológico $(\mathrm{p}=0.004)$ y desarrollo de complicaciones neurológicas postquirúrgicas $(\mathrm{p}=0.012)$. Para cada uno de los factores se realizó análisis univariante correlacionándolo con el resultado quirúrgico, el estado neurológico postquirúrgico (escala de Nurick y de la JOA) y la razón de recuperación de Hirabayashi.

\section{A) Edad}

Los pacientes que mejoraron tras la cirugía eran una década más jóvenes que los que no mejoraron o empeoraron (47.00 años vs. 56.58 años), según confirmó la t de Student $(\mathrm{p}=0.008)$. Para realizar una estimación del riesgo se agrupó a los pacientes en dos grupos de edad con un punto de corte establecido en los 45 años. El 81\% de los pacientes menores de 45 años se benefició de la cirugía frente al $53.1 \%$ de los mayores de 45 años, con una $p=0.046$ en la prueba exacta de Fisher, siendo la odds ratio de 3.75 (intervalo de confianza del $95 \%=1.03-13.64$ ) (figura 10). Igualmente la razón de recuperación de los pacientes menores de 45 años fue significativamente mayor que la de los pacientes mayores de 45 años (50.05 vs 20.65) con una $\mathrm{p}=0.033$ en la $\mathrm{t}$ de Student (figura 11).

La comparación de las medias de edad de los distintos grados de afectación neurológica tras la cirugía utilizando la escala de Nurick se quedó al borde de la significación estadística (test ANOVA, $\mathrm{p}=0.058$ ), y la correlación entre la edad y la puntuación postquirúrgica en la escala de la JOA fue baja con una $r$ de Pearson de $-0.352(\mathrm{p}=0.01)$.

\section{B) Factores de riesgo vascular}

El 68.8\% de los pacientes sin factores de riesgo vascular mejoraron tras la cirugía, mientras que únicamente el 20\% de los que los presentaban lo hicieron (figura 10). El test exacto de Fisher mostró una $\mathrm{p}=0.05$, en el límite exacto de la significación estadística, siendo la odds ratio de 8.80 (intervalo de confianza del 95\% $=0.99-85.58$ ). La razón de recuperación de los pacientes con factores de riesgo vascular era significativamente menor que la de los pacientes $\sin$ factores de riesgo vascular (-15.24 vs 37.25$)$ con una $\mathrm{p}=0.022$ en la $t$ de Student (figura 11).

$\mathrm{Al}$ enfrentar los factores de riesgo vascular con la escala de Nurick postoperatoria, el test $\chi^{2}$ de Pearson con corrección iterativa de Montecarlo volvió a encontrarse al límite de la significación $(\mathrm{p}=0.055)$. Sin embargo, la puntuación media postoperatoria en la escala de la JOA en los pacientes con factores de riesgo vascular era significativamente menor que en los pacientes sin ellos (10.60 vs 14.02 , $t$ de Student $\mathrm{p}=0.022$ ) (figura 12).

\section{C) Tiempo de evolución}

El tiempo de evolución de los pacientes con mal resultado quirúrgico triplicaba al de los pacientes con buen resultado (36.69 vs 11.26 meses), con una $\mathrm{p}=0.002$ para la prueba $t$ de Student. Para realizar la estimación de riesgo se agrupó a los pacientes tomando como punto de corte 12 meses de evolución. El test $\chi^{2}$ de Pearson corroboró que los pacientes con un periodo sintomático inferior al año se beneficiaban de la cirugía con mayor frecuencia que los pacientes que habían presentado síntomas por más de un año $(80.0 \%$ vs $33.3 \%, \mathrm{p}=0.001)$, con una odds ratio de 8.00 (intervalo de confianza del 95\% $=2.22-28.86$ ) (figura 10). Los pacientes con evolución menor de un año presentaban razones de recuperación significativamente mayores que los pacientes con clínica de duración superior al año (42.80 vs 11.88 ) con una $\mathrm{p}=0.030$ en la $\mathrm{t}$ de Student (figura 11).

\section{D) Estado neurológico prequirúrgico}

La puntuación prequirúrgica en la escala de la JOA de los pacientes que se beneficiaron de la cirugía fue significativamente mayor que la de los que no lo hicieron (13.71 vs 11.32 , t de Student $\mathrm{p}<0.001$ ), obteniéndose una alta correlación entre las puntuaciones pre y postoperatorias en dicha escala, con una $r$ de Pearson de $0.761(p<0.001)$ (figura 13), y entre la puntuación preoperatoria y la razón de recuperación ( $\mathrm{r}$ de Pearson=0.465, $\mathrm{p}<0.001$ ) (figura 14). En la tabla 10 se tabulan los grados pre y postquirúrgicos de la escala de Nurick. El test $\chi^{2}$ de Pearson con corrección iterativa de Montecarlo determinó la existencia de diferencias estadísticamente significativas en el resultado quirúr- 
Martín y col

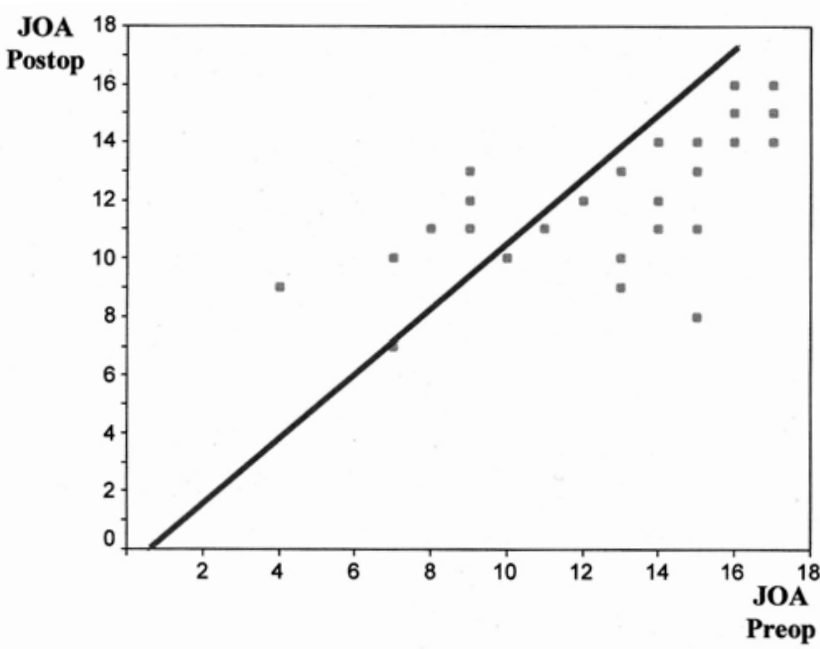

Figura 13. La correlación entre el estado neurológico pre y postquirúrgico según la escala de la asociación de ortopedia japonesa (JOA) resultó elevada ( $r$ de Pearson=0.761).

gico de los diferentes grupos $(\mathrm{p}<0.001)$, encontrándose $-\mathrm{al}$ igual que ocurrió con la escala de la JOA- una correlación elevada entre las puntuaciones pre y postoperatorias ( $\mathrm{r}$ de Pearson $=0.774, \mathrm{p}<0.001)$. El test ANOVA también detectó diferencias entre las razones de recuperación medias de los diferentes grupos de la escala de Nurick $(\mathrm{p}<0.001)$. Para realizar la estimación del riesgo agrupamos los pacientes según el estado neurológico preoperatorio en grados 1 y 2 de Nurick (leve) y grado 3, 4 ó 5 (moderado-grave). El $93.1 \%$ de los pacientes con mielopatía grado 1 y 2 de Nurick se beneficiaron de la cirugía, frente $29.7 \%$ de los enfermos con mielopatía grado 3, 4 ó 5 (test exacto de Fisher, $\mathrm{p}<0.001)$. La estimación de riesgo arrojó una odds ratio
Neurocirugía $2005 ; 16: 235-255$

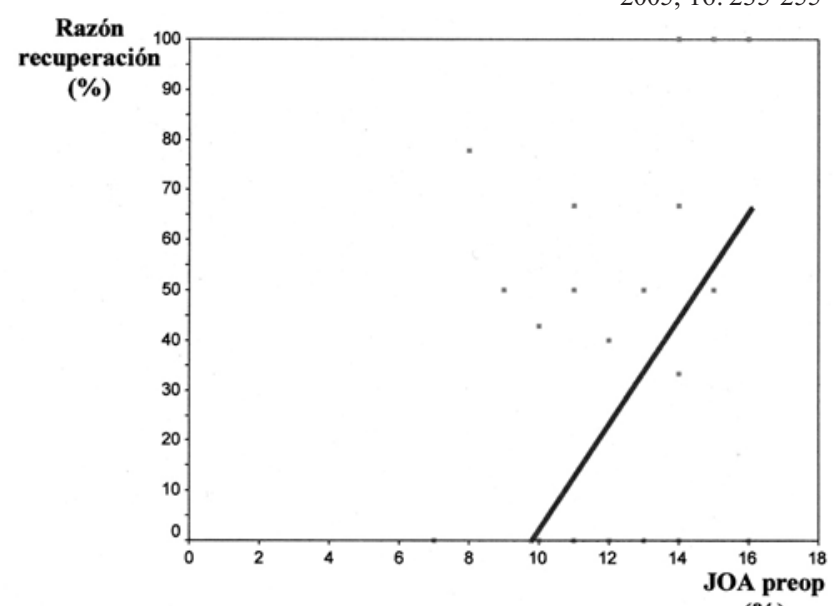

(\%)

Figura 14. La correlación entre el estado neurológico prequirúrgico cuantificado con la escala de la asociación de ortopedia japonesa (JOA) y la razón de recuperación de Hirabayashi fue de intensidad moderada ( $r$ de Pearson=0.465).

de 32.79 (intervalo de confianza del 95\%=6.08-176.72) (figura 10). La razón de recuperación de los pacientes en grados preoperatorios 1 y 2 de Nurick fue mayor que la de los pacientes con grados preoperatorios 3, 4 y 5 (57.47 vs 1.89) con una $\mathrm{p}<0.001$ en la t de Student (figura 11).

\section{E) Diagnóstico neurorradiológico}

El $82.6 \%$ de los pacientes diagnosticados de herniación discal cervical mejoraron tras la cirugía, frente al 50.0\% de los pacientes con diagnóstico de espondilosis (test exacto de Fisher, $\mathrm{p}=0.021$ ), con una odds ratio de 4.75 (intervalo de confianza del 95\%=1.30-17.33) (figura 10). La razón de recuperación de los pacientes diagnosticados de hernia

Tabla 10

Nurick preoperatorio vs Nurick postoperatorio

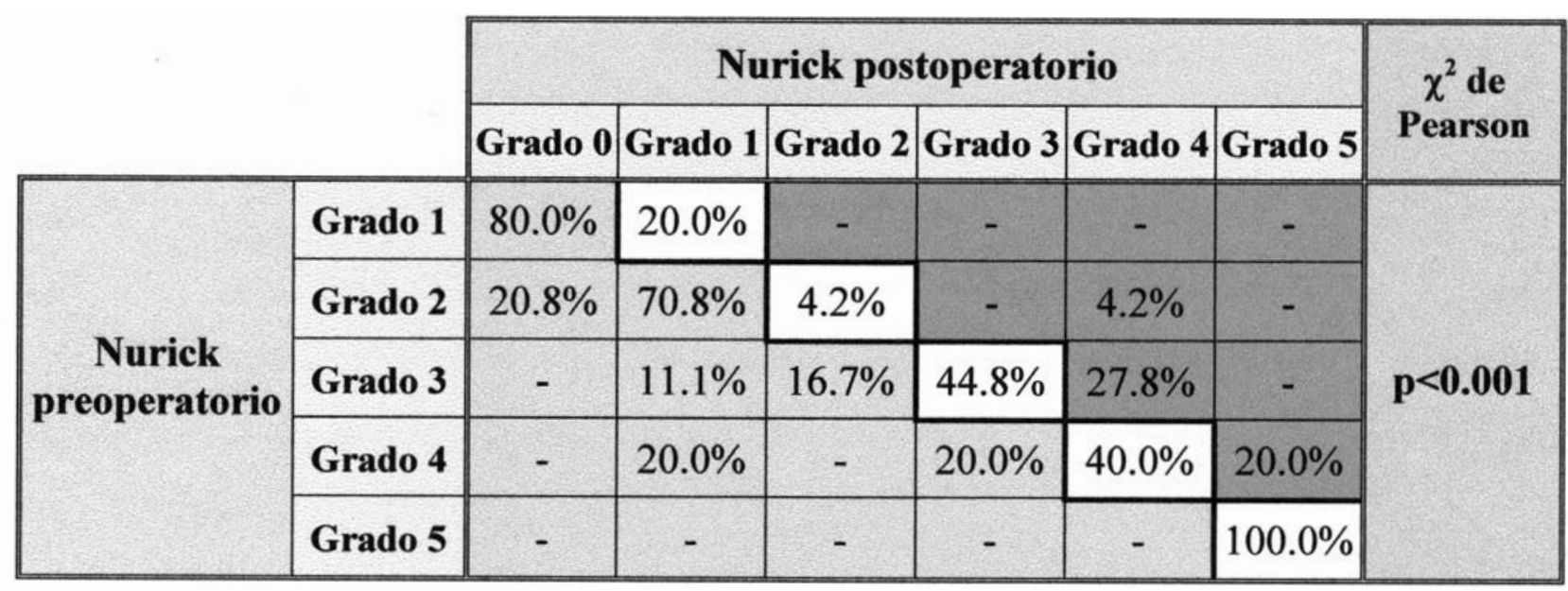


discal blanda resultó significativamente mayor que la de los pacientes diagnosticados de espondilosis (46.01 vs 21.79) con una $\mathrm{p}=0.046$ en la $t$ de Student (figura 11).

La tabulación del diagnóstico neurorradiológico con respecto al resultado clínico según la escala de Nurick se quedó al límite de la significación estadística (test $\chi^{2}$ de Pearson con corrección iterativa de Montecarlo, $\mathrm{p}=0.057)$. Sin embargo, la puntuación postoperatoria en la escala de la JOA de los pacientes con diagnóstico de hernia discal era significativamente mayor que la de los pacientes diagnosticados de espondilosis (14.91 vs 12.77 , $\mathrm{p}=0.014$ )

\section{F) Presencia de hiperseñal intramedular en la resonancia magnética}

La comparación de la clínica neurológica postoperatoria según la escala de Nurick mediante la prueba $\chi^{2}$ de Pearson con corrección iterativa de Montecarlo mostró diferencias estadísticamente significativas entre los pacientes que presentaban hiperseñal intramedular en las secuencias potenciadas en $\mathrm{T} 2$ en la resonancia magnética y aquéllos que no la presentaban ( $\mathrm{p}=0.002$ ). Asimismo, la puntuación postoperatoria en la escala de la JOA de los pacientes con hiperseñal intramedular era significativamente menor que la de los pacientes con señal normal (12.79 vs 16.70, t de Student $\mathrm{p}=0.001$ ). Mientras que $100 \%$ de los pacientes $\sin$ alteración de la señal medular mejoraron tras la cirugía, solamente el $54.2 \%$ de los que presentaban hiperseñal intramedular lo hizo (test exacto de Fisher, $\mathrm{p}=0.014$ ). La estimación de riesgo arrojó una odds ratio de 1.85 (intervalo de confianza del $95 \%=1.28$-2.67) (figura 10). La razón de recuperación de los pacientes con señal medular alterada fue menor que la de los pacientes con señal medular normal (17.81 vs 86.67 , t de Student $\mathrm{p}<0.001)$ (figura 11).

\section{G) Alteración de la conducción medular en el estudio neurofisiológico}

En conjunto, el 100\% de los pacientes con conducción medular normal se beneficiaron de la intervención quirúrgica, mientras que solamente mejoró una tercera parte de los que tenían la conducción medular alterada (test exacto de Fisher, $p=0.012$ ). La odds ratio fue de 3.00 (intervalo de confianza del 95\%=1.47-6.14) (figura 10). La razón de recuperación de los pacientes con estudio de conducción medular normal fue mayor que la de los pacientes con conducción alterada (55.56 vs 11.56) con una $\mathrm{p}=0.048$ en la $\mathrm{t}$ de Student (figura 11).

La prueba $t$ de Student demostró que los pacientes con un estudio neurofisiológico de conducción medular normal tenían puntuaciones postoperatorias en la escala de la JOA significativamente más altas que aquéllos que presentaban alteraciones en los potenciales evocados somatosensoria- les o en la estimulación magnética transcraneal (16.17 vs 12.00, $\mathrm{p}<0.001)$. Sin embargo, la prueba $\chi^{2}$ de Pearson con corrección iterativa de Montecarlo no alcanzó la significación estadística al tabular la situación neurológica postquirúrgica según la escala de Nurick con los hallazgos del estudio neurofisiológico ( $\mathrm{p}=0.080$ ), de nuevo probablemente por el bajo número de pacientes incluidos.

\section{H) Complicaciones neurológicas}

Únicamente el $30.0 \%$ de los enfermos que padeció una complicación neurológica mejoró tras la cirugía, frente al $72.1 \%$ de los pacientes sin este tipo de complicación (test exacto de Fisher, $\mathrm{p}=0.025$ ). Se obtuvo una odds ratio de 6.03 (intervalo de confianza del $95 \%=1.33-27.22$ ) (figura 10). Los pacientes que presentaron una complicación neurológica presentaban razones de recuperación significativamente inferiores que aquéllos sin complicaciones (-23.62 vs 45.30, t de Student $\mathrm{p}<0.001)$ (figura 11). El test $\chi^{2}$ de Pearson con corrección iterativa de Montecarlo mostró la existencia de diferencias estadísticamente significativas en la escala de Nurick postoperatoria entre los enfermos que habían sufrido una complicación neurológica y los que no habían tenido complicaciones o éstas eran de tipo no neurológico $(\mathrm{p}=0.005)$. Igualmente, la puntuación postoperatoria en la escala de la JOA fue menor en los pacientes con complicaciones neurológicas con respecto al resto de los enfermos (14.28 vs 11.20 , $t$ de Student $\mathrm{p}=0.005$ ).

En este punto resulta conveniente realizar dos consideraciones. De los pacientes que presentaron como complicación un agravamiento de la mielopatía, solamente el $16.7 \%$ se benefició a largo plazo de la cirugía (test exacto de Fisher, $\mathrm{p}=0.006$ ), siendo la razón de recuperación de los pacientes que presentaron esta complicación inferior que la de los pacientes que no la presentaron (-32.22 vs 40.54, $\mathrm{t}$ de Student $\mathrm{p}<0.001)$. Cuando el deterioro neurológico, fuese del tipo que fuese, se prolongó por más de tres meses ningún paciente logró buen resultado a largo plazo (test exacto de Fisher, $\mathrm{p}=0.004$ ), siendo la razón de recuperación de estos pacientes significativamente menor $(-57.24$ vs 41.63 , $\mathrm{t}$ de Student $\mathrm{p}<0.001$ ).

\section{Reintervenciones}

Un 3.7\% de los pacientes ( $\mathrm{n}=2$ ) fueron reintervenidos. En el primero de ellos se indicó la cirugía sobre el mismo espacio a los 2 meses de la primera intervención como consecuencia de la claudicación del injerto óseo. El otro presentó deterioro neurológico lentamente progresivo tras haber mostrado mejoría inicial, siendo intervenido del espacio inferior al operado inicialmente como consecuencia de la acentuación de los cambios degenerativos, nuevamente por vía anterior. En ambos casos el resultado quirúrgico fue bueno, manteniéndose a largo plazo. 


\section{Discusión}

Esta serie, aunque cuenta con pocos pacientes, es superponible en cuanto a características epidemiológicas, clínicas y radiológicas al resto de las series de la literatura, y se corresponde con la imagen clásica que se describe en la práctica totalidad de los artículos de revisión publicados sobre el tema ${ }^{7,13,14,17,32,38,39,40,57,54,59,73}$. El paciente tipo es un varón de 50-60 años, que muestra clínica de aproximadamente un año y medio de evolución compatible con un síndrome cordonal anterior de intensidad moderada. Cabe destacar que en un $25 \%$ de los casos la consulta especializada se difiere más de dos años, lo que refleja la dificultad existente en realizar un correcto diagnóstico de una patología que afecta a pacientes de edad avanzada, se manifiesta fundamentalmente como una alteración de la marcha, y que por tanto puede ser interpretada como la marcha fisiológica del anciano.

Tal y como ha sido referido por otros autores, los pacientes en los que se descubre una hernia discal como causante de mielopatía son más jóvenes, se presentan con mayor frecuencia en forma de mielorradiculopatía y su cuadro clínico usualmente es menos acusado y de menor duración, correspondiéndose con bastante asiduidad con un síndrome de hemisección medular ${ }^{10,42,61}$. Los pacientes mayores suelen padecer una discartrosis multinivel y presentar clínica de mayor gravedad consecuente con un síndrome cordonal anterior o un síndrome centromedular ${ }^{51}$.

En las extremidades superiores los grupos musculares más afectados son la musculatura intrínseca de la mano $(56.6 \%)$ y el triceps $(28.9 \%)$, mientras que en las extremidades inferiores son ileopsoas $(38.8 \%)$ y cuadriceps $(26.3 \%)^{12}$. Sin embargo, parece ser que la espasticidad es el eje sobre el que se desarrolla la disfunción muscular responsable de la alteración de la marcha, ya que ésta se encuentra presente en más del $80 \%$ de los pacientes, mientras que menos de la mitad presentan un déficit motor en la musculatura proximal de las extremidades inferiores ${ }^{7,12,13,39,57}$. De hecho, en el estudio de Chiles y cols la espasticidad causaba una gran parte del trastorno de la marcha tanto preoperatorio como postoperatorio ${ }^{12}$. La alteración de esfínteres y la disfunción sexual son relativamente infrecuentes y aparecen en casos avanzados ${ }^{7,13,39,57}$.

Los resultados a largo plazo de la discectomía y fusión intersomática por vía anterior para el tratamiento de la mielopatía cervical son variables, con cifras de mejoría en torno al $50 \%$ de los pacientes en algunas publicaciones mientras que en otras los buenos resultados superan el $90 \%$ de los $\operatorname{casos}^{5,14,17,22,26,32,40,55,54,59,60,73,76}$. Es posible que en estos dispares resultados influya tanto la técnica quirúrgica -que se consiga un mayor grado de descompresión del cordón medular- como la selección de los pacientes. Desde el punto de vista quirúrgico, las diferentes técnicas de discectomía y fusión intersomática por vía anterior resultan muy similares en cuanto a concepción, pero muy diferentes en cuanto a su capacidad para descomprimir las estructuras mielorradiculares. Mientras que en la técnica de Cloward el espacio se encuentra limitado al cilindro que talla el perforador en el espacio intervertebral, en la corpectomía el fresado puede extenderse tanto lateralmente como en el eje vertical hasta que el cirujano se encuentre plenamente seguro de que la médula ha sido descomprimida en su totalidad. Con toda probabilidad, la elección de la técnica quirúrgica a emplear, tras una valoración clínico-radiológica individualizada, redundaría en un mejor resultado de la cirugía: mientras que la mielopatía secundaria a una hernia discal blanda en un paciente joven podría tratarse mediante una discectomía simple, la patología cervicoartrósica multiespacio en un enfermo de mayor edad seguramente se beneficiaría más de una vertebrectomía con fusión intersomática instrumentada.

Diversos estudios han intentado determinar qué factores presentan valor pronóstico sobre el resultado de la cirugía en la mielopatía cervical espondilótica, sin que exista una total congruencia entre ellos. La gravedad inicial de la clínica, 5,12,17,32,38,40,48,57,59,60 y su duración ${ }^{6,15,19,22,23,26,35,37,40,45,48,55,57,72,73}$, la edad del paciente $e^{6,8,19,26,35,40,48,50,57}$, el tipo de patología causante del cuadro (hernia discal blanda o discartrosis) $)^{12,26,36,40,76}$, la presencia de hiperseñal medular ${ }^{12,24,35,43,46,48,50,52,65} \mathrm{o}$ atrofia del cordón medular en la resonancia magnética ${ }^{12,19,35,37,45,48,57,72}$, el diámetro del canal raquídeo ${ }^{40,63}$, la actitud estática del raquis cervical (cifosis o lordosis) ${ }^{35,50}$, el deterioro de la conducción medular en el estudio neurofisiológico ${ }^{2,3,10,44,62,67}$, el número de niveles intervenido ${ }^{8,12,19,57,72}$, las complicaciones quirúrgicas ${ }^{38,76}$ y la asociación de otras patologías médicas de carácter grave ${ }^{34,38,40}$, se han propuesto como posibles factores pronósticos. El presente estudio parece haber confirmado algunos de ellos, aunque los resultados deben tomarse con precaución dada la retrospectividad del estudio y el limitado número de pacientes analizado.

La clínica del paciente es uno de los factores pronósticos mejor establecidos en la literatura, aunque no todos los estudios muestran unanimidad en cuanto a qué aspecto de ésta es el más importante. La gravedad de ésta parece relacionarse estrechamente tanto con el estado neurológico postquirúrgico como con la razón de recuperación en múltiples trabajos ${ }^{5,8,12,14,17,32,38,40,48,57,59,60}$, aunque en otros no se ha confirmado como un factor pronóstico relevante ${ }^{6,15,19,26,35,50,72,73,76}$. En nuestros pacientes parece existir una fuerte correlación entre el estado neurológico prequirúrgico, el postquirúrgico y el grado de recuperación, beneficiándose las mielopatías leves claramente más que las graves. Sin embargo -y a la vista de los estudios prospectivos de Sampath ${ }^{58}$ y Kadanka ${ }^{33}$, de los que parece desprenderse la falta de superioridad de la 
cirugía sobre el tratamiento conservador en la mielopatía de gravedad leve o mederada- hay que ser extremadamente cauto a la hora de interpretar estos resultados que, en ausencia de un grupo control, podrían ser atribuidos a una mejoría espontánea de la enfermedad. También existe tendencia a aceptar que aquellos pacientes cuya evolución sintomática supera el año obtienen menor beneficio de la cirugía $^{6,15,19,22,23,26,35,37,40,45,48,55,57,72,73}$, a pesar de que en otros estudios no se han detectado diferencias entre los pacientes con clínica de corta y larga duración ${ }^{20,32,38,50,76}$. En este punto los resultados de nuestro análisis estadístico resultan paradójicos, ya que la evolución sintomática sí parece relacionarse con la razón de recuperación, pero no con el estado neurológico postquirúrgico. Aunque inicialmente se intentó relacionar el pronóstico funcional y el síndrome medular con el que debutaba el paciente, actualmente parece existir evidencia suficiente como para rebatir esta teoría ${ }^{36,76}$.

Potencialmente, la edad del paciente puede intervenir de diversas formas en el resultado quirúrgico, y así parece demostrarse en diversos estudios $\mathrm{s}^{6,8,19,26,35,40,48,50,57}$. Los enfermos de edad avanzada suelen tener, con mayor frecuencia, otras patologías concomitantes que podrían ensombrecer el pronóstico funcional: presentan habitualmente enfermedad espondilótica y, además, ésta suele ser multinivel ${ }^{51}$. Sin embargo, otros autores no han conseguido demostrar que los pacientes de mayor edad tengan un peor pronóstico ${ }^{12,15,32,73,76}$, y algunos trabajos parecen poner de manifiesto la efectividad de la cirugía descompresiva en los ancianos afectos de mielopatía cervical espondilótica, si la indicación es adecuada ${ }^{51,66}$. En cualquiera de los casos, no se ha establecido un punto de corte a partir del cual el pronóstico empeore claramente, y los diferentes trabajos sitúan la edad a partir de la que el beneficio de la cirugía decrece significativamente en 40, 60 ó 70 años. Este punto de corte tiene un poco de arbitrario, ya que probablemente está más relacionado con la potencia estadística de los estudios que con un cambio pronóstico real. Las series que, como la nuestra, cuentan con un número limitado de pacientes, tienen más probabilidad de dar un resultado no significativo o de que, si se alcanza la significación estadística, el punto de corte se encuentre en edades bajas. Así, en nuestro estudio, el corte se localizó en 45 años demostrándose que los pacientes menores de esta edad presentaban razones de recuperación más elevadas, y existiendo una correlación de intensidad leve-moderada entre la edad y la puntuación JOA postquirúrgica.

El estudio iconográfico, además de aportar información para la planificación quirúrgica, también contribuye a establecer un pronóstico funcional para estos pacientes. Probablemente los dos factores que se cree presentan mayor repercusión sobre el resultado final de la cirugía sean las anomalías de señal intramedular en la resonancia magnética y el área medular. La presencia de hiperseñal intramedular en las secuencias de resonancia ponderadas en $\mathrm{T} 2$ es un punto actual de controversia para muchos autores, ya que algunos estudios han mostrado un mal resultado cuando ésta existe ${ }^{12,24,35,43,46,52,65}$; en otros no se han observado diferencias ${ }^{49,70}$, y alguna serie reciente objetiva un mejor resultado cuando existe hiperseñal ${ }^{64}$. El incremento de la intensidad de la señal en las secuencias ponderadas en $\mathrm{T} 2$ puede representar un amplio espectro de cambios patológicos del cordón medular, que van desde el edema medular hasta la mielomalacia, pasando por diferentes grados de gliosis, inflamación o isquemia ${ }^{46}$. En aquellos casos en los que la hiperseñal representase edema o inflamación el paciente tendría mayor probabilidad de beneficiarse de la cirugía, mientras que en los que tradujese mielomalacia la posibilidad de una mejoría sería escasa. Los trabajos publicados prácticamente de forma simultánea por Morio y cols $^{48}$ y Chen y cols ${ }^{11}$ parecen clarificar esta situación. Las áreas de hiperintensidad en T2, de borde impreciso, y que no se asocian a hiposeñal en las secuencias en T1 (tipo 1 de Chen), probablemente se corresponden a edema o inflamación medular, y se relacionan con una buena respuesta a la cirugía. Las áreas de hiperintensidad en T2, de borde bien delimitado, y que se asocian a hiposeñal en las secuencias en T1 (tipo 2 de Chen), probablemente se corresponden a mielomalacia o gliosis intensa, y se relacionan con una mala respuesta al tratamiento quirúrgico ${ }^{11,48}$. Por otra parte, es una práctica ampliamente extendida tratar con corticoides a los pacientes que presentan una mielopatía de rápida evolución, resultando verosimil que el sustrato patológico de la hiperseñal medular sea edema en aquéllos que respondan favorablemente a este tratamiento. En nuestro estudio la totalidad de los pacientes sin alteración de la señal medular mejoraron tras la cirugía, con una razón de recuperación superior al $80 \%$.

El grado de compresión medular, definido por el área medular en un corte axial de resonancia magnética o de mielo-TC o por la razón de compresión antero-posterior, parece ser uno de los factores más fiables a la hora de predecir el pronóstico funcional de un determinado paciente, y parece que áreas inferiores a los $30-40 \mathrm{~mm}^{2}$ o razones de compresión inferiores al $20 \%$ se asocian con un mal resultado y un pésimo pronóstico ${ }^{12,19,35,37,45,48,57,72}$. Sin embargo, de nuevo existen estudios que no refrendan estos hallaz$\operatorname{gos}^{17,76}$

Otro factor radiológico, que parece relacionarse con la respuesta del paciente a la cirugía, es el diagnóstico patológico, confirmándose que la herniación del núcleo pulposo se comporta de forma radicalmente diferente a la enfermedad espondilótica degenerativa ${ }^{12,26,40,42,61}$. En nuestra serie, el $80 \%$ de los pacientes intervenidos como consecuencia de una hernia discal blanda mostró mejoría, con razones de recuperación próximas al 50\%. Hay que tener en cuenta que los cambios espondilóticos suelen ser multisegmentarios, $\mathrm{y}$ 
habiéndose relacionado la patología multinivel ${ }^{8,12,19,57,72}$ y la pérdida de la lordosis cervical ${ }^{35,50}$ con una peor respuesta al tratamiento, estas situaciones podrían potenciarse o actuar como factores de confusión. Por otra parte, cuando nos enfrentamos a un paciente de edad avanzada con una espondilosis multiespacio, hay que cerciorarse de realizar una descompresión suficientemente amplia, ya que un porcentaje no despreciable de los fracasos quirúrgicos en este tipo de patología se deben a una descompresión inadecuada de las estructuras mielorradiculares. También habría que pensar qué porcentaje de estos pacientes no mejora como consecuencia de la elección de una vía inadecuada, indicando abordajes posteriores en pacientes con deformidad cifótica.

La utilidad de los potenciales evocados somatosensoriales y de la estimulación magnética transcraneal como apoyo diagnóstico en la mielopatía cervical espondilótica se encuentra ampliamente demostrada. La sensibilidad de estas técnicas ronda el 95\% (93\%-100\%) para la estimulación magnética transcraneal y el 70\% (43\%-100\%) para los potenciales evocados somatosensoriales ${ }^{3,10,25,69}$. Sin embargo, su capacidad para predecir el resultado quirúrgico en un determinado paciente se encuentra en entredicho, si bien es cierto que aquellos casos con una afectación moderada-grave de estos estudios la mejoría, tanto neurofisiológica como clínica, tiende a ser pobre $2,3,10,44,62,67$. El único parámetro que parece relacionarse con un buen pronóstico tras la cirugía es la alteración aislada del componente N13 de los potenciales somatosensoriales, que representaría un signo de daño menor, y potencialmente reversible, del asta posterior ${ }^{3,56}$. En la presente serie todos los pacientes que no presentaban alteraciones de la conducción medular se beneficiaron de la cirugía con una razón de recuperación superior al 50\%, hallazgo que resulta congruente con la tendencia a mostrar mejoría de aquellos pacientes con una mielopatía de intensidad leve.

El papel de otras patologías intercurrentes en el pronóstico funcional de la mielopatía cervical espondilótica ha sido tenido en cuenta con anterioridad $\mathrm{d}^{34,38,40} \mathrm{y}$, de entre estos, los factores vasculares se han implicado en el desarrollo fisiopatológico de la mielopatía en múltiples estudios ${ }^{1,9,30,68}$. En una publicación previa relacionamos los factores de riesgo vascular (hipertensión arterial, hipercolesterolemia, hábito tabáquico, diabetes, consumo excesivo de alcohol) con la posibilidad de desarrollar complicaciones neurológicas en la cirugía del raquis cervical por vía anterior, situación que parece confirmarse para el subgrupo de pacientes afectos de mielopatía cervical espondilótica en el presente estudio ${ }^{41}$. En esta serie, los pacientes con factores de riesgo vascular han presentado una baja probabilidad de beneficiarse de la cirugía, mostrando una razón de recuperación menor y un peor estado neurológico postquirúrgico. Igualmente, el reciente trabajo de Kawaguchi y cols ha relacionado los niveles elevados de hemoglobina glicosilada -que indican una diabetes mal controlada- con una mala respuesta al tratamiento quirúrgico ${ }^{34}$. En nuestra opinión estos hallazgos justifican la realización de un estudio prospectivo para comprobar la fidelidad de los resultados y un manejo agresivo de los factores sistémicos que podrían interferir en el resultado de la intervención.

De todos los factores que podrían intervenir en el resultado clínico de los pacientes el único prevenible son las complicaciones quirúrgicas ${ }^{38,76}$. El deterioro postquirúrgico de un paciente afecto de mielopatía es una complicación temida por todo cirujano y prácticamente imposibilita la consecución de un buen resultado clínico a largo plazo. Debemos remarcar que en esta serie las complicaciones neurológicas se encontraron relacionadas con la presencia de factores vasculares: la totalidad de los pacientes con factores de riesgo vascular tuvo algún tipo de complicación, correspondiendo el $80 \%$ a complicaciones neurológicas, y siendo el $60 \%$ permanentes. Esto nos hace recomendar un exquisito cuidado a la hora de planificar y llevar a cabo la cirugía en este tipo de pacientes.

\section{Conclusiones}

La discectomía y artrodesis intersomática por vía anterior mediante técnica de Cloward se ha mostrado efectiva en el tratamiento de la mielopatía cervical espondilótica, aunque actualmente existe un subgrupo de pacientes en los que probablemente no debería considerarse como procedimiento de elección. Aquellos enfermos afectos de espondiloartrosis multiespacio, especialmente si se asocia una estenosis del canal cervical, probablemente se beneficiarían de abordajes posteriores si la lordosis cervical se encuentra conservada o de procedimientos anteriores más agresivos, como la corpectomía.

Este estudio parece correlacionar con una mala respuesta a la cirugía la edad avanzada, la mielopatía de intensidad grave y de larga evolución, los factores de riesgo vascular, la espondilosis multinivel, la presencia de hiperseñal intramedular en la resonancia magnética ponderada en T2, la alteración de la conducción medular en los estudios neurofisiológicos y el desarrollo de complicaciones neurológicas. Con estos resultados en la mano creemos que la cirugía en los pacientes con mielopatía cervical espondilótica debe indicarse de forma precoz en la evolución de la enfermedad, aunque las discrepancias objetivadas en la literatura únicamente podrán aclararse tras la realización de estudios prospectivos y randomizados, cuyos resultados nos devolverán con toda probabilidad una imagen más clara del tipo de paciente subsidiario de tratamiento quirúrgico y del momento en el que indicar dicho tratamiento. 


\section{Bibliografía}

1. al Mefty, O., Harkey, H.L., Marawi, I., Haines, D.E., Peeler, D.F., Wilner, H.I., et al.: Experimental chronic compressive cervical myelopathy. Surg Radiol Anat. 1993; 79: 550-561.

2. Baba, H., Maezawa, Y., Imura, S., Kawahara, N., Tomita, K.: Spinal cord evoked potential monitoring for cervical and thoracic compressive myelopathy. Paraplegia 1996; 34: 100-106.

3. Bednarik, J., Kadanka, Z., Vohanka, S., Stejskal, L., Vlach, O., Schroder, R.: The value of somatosensory- and motor-evoked potentials in predicting and monitoring the effect of therapy in spondylotic cervical myelopathy. Prospective randomized study. Spine 1999; 24: 1593-1598.

4. Benzel, E.C., Lancon, J., Kesterson, L., Hadden, T.: Cervical laminectomy and dentate ligament section for cervical spondylotic myelopathy. J Spinal Disord 1991; 4: 286-295.

5. Bernard, T.N.J., Whitecloud, T.S.I.: Cervical spondylotic myelopathy and myelorradiculopathy: anterior decompression and stabilization with autogenous fibula strut graft. Clin Orthop 1987; 221: 149-157.

6. Bertalanffy, H., Eggert, H.R.: Clinical long-term results of anterior discectomy without fusion for treatment of cervical radiculopathy and myelopathy. Acta Neurochir (Wien) 1988; 90: 127-135.

7. Bohlman, H.H.: Cervical spondylosis and myelopathy. Instr Course Lect 1995; 44: 81-97.

8. Brandt, R.A., Fager, C.A.: Cervical spondylosis. Prognostic value of preoperative signs and symptoms. Arq Neuropsiquiatr. 1976; 34: 32-39.

9. Carlson, G.D., Warden, K.E., Barbeau, J.M., Bahniuk, E., Kutina-Nelson, K.L., Biro, C.L., et al.: Viscoelastic relaxation and regional blood flow response to spinal cord compression and decompression. Spine 1997; 22: 1285-1291.

10. Chang, C.W., Lin, S.M.: Predictability of surgical results of herniated disc-induced cervical myelopathy based on spinal cord motor conduction study. Neurosurg Rev 1999; 22: $107-111$.

11. Chen, C.J., Lyu, R.K., Lee, S.T., Wong, Y.C., Wang, L.J.: Intramedullary high signal intensity on T2-weighted MR images in cervical spondylotic myelopathy: prediction of prognosis with type of intensity. Radiology 2001; 221: 789794.

12. Chiles, B.W., 3d, Leonard, M.A., Choudhri, H.F., Cooper, P.R.: Cervical spondylotic myelopathy: patterns of neurological deficit and recovery after anterior cervical decompression. Neurosurgery 1999; 44: 762-770.

13. Clark, C.R.: Cervical spondylotic myelopathy: History and physical findings. Spine 1988; 13: 847-849.

14. Clavel-Escribano, M., Clavel-Laria, P.: Tratamiento quirúrgico de la mielopatía cervical espondiloartrósica. Neurocirugia 1996; 7: 4-10.
15. Ebersold, M.J., Pare, M.C., Quast, L.M.: Surgical treatment for cervical spondylitic myelopathy. Surg Radiol Anat. 1995; 82: 745-751.

16. Edwards, C.C., Heller, J.G., Murakami, H.: Corpectomy versus laminoplasty for multilevel cervical myelopathy: an independent matched-cohort analysis. Spine 2002; 27: $1168-1175$.

17. Emery, S.E., Bohlman, H.H., Bolesta, M.J., Jones, P.K.: Anterior cervical decompression and arthrodesis for the treatment of cervical spondylotic myelopathy. Two to seventeen-year follow-up. J Bone Joint Surg Am 1998; 80: 941-951.

18. Fouyas, I.P., Statham, P.F., Sandercock, P.A., Lynch, C.: Surgery for cervical radiculomyelopathy (Cochrane Review). Cochrane. Database. Syst. Rev 2001; 3: CD001466.

19. Fujiwara, K., Yonenobu, K., Ebara, S., Yamashita, K., Ono, K.: The prognosis of surgery for cervical compression myelopathy. An analysis of the factors involved. J Bone Joint Surg Br 1989; 71: 393-398.

20. Gonzalez-Feria, L.: The effect of surgical immobilization after laminectomy in the treatment of advanced cases of cervical spondylotic myelopathy. Acta Neurochir (Wien) 1975; 31: 185-193.

21. Gross, J.D., Benzel, E.C.: Dorsal surgical approach for cervical spondylotic myelopathy. Tech Neurosurg 1999; 5: 162-176.

22. Guidetti, B., Fortuna, A.: Long-term results of surgical treatment of myelopathy due to cervical spondylosis. Surg Radiol Anat. 1969; 30: 714-721.

23. Hamanishi, C., Tanaka, S.: Bilateral multilevel laminectomy with or without posterolateral fusion for cervical spondylotic myelopathy: relationship to type of onset and time until operation. Surg Radiol Anat. 1996; 85: 447-451.

24. Harada, A., Mimatsu, K.: Postoperative changes in the spinal cord in cervical myelopathy demonstrated by magnetic resonance imaging. Spine 1992; 17: 1275-1280.

25. Hayashida, T., Ogura, T., Hase, H., Osawa, T., Hirasawa, Y.: Estimation of cervical cord dysfunction by somatosensory evoked potentials. Muscle Nerve 2000; 23: 1589-1593.

26. Heidecke, V., Rainov, N.G., Marx, T., Burket, W.: Outcome in Cloward anterior fusion for degenerative cervical spine disease. Acta Neurochir (Wien) 2000; 142: 283-291.

27. Heller, J.G., Edwards, C.C., Murakami, H., Rodts, G.E.: Laminoplasty versus laminectomy and fusion for multilevel cervical myelopathy: an independent matched cohort analysis. Spine 2001; 26: 1330-1336.

28. Hirabayashi, K., Bohlman, H.H.: Multilevel cervical spondylosis. Laminoplasty versus anterior decompression. Spine 1995; 20: 1732-1734.

29. Hirabayashi, K., Miyakawa, J., Satomi, K., Maruyama, T., Wakano, K.: Operative results and postoperative progression of ossification among patients with ossification of cervi- 
cal posterior longitudinal ligament. Spine 1981; 6: 354-364.

30. Hoff, J.T., Nishimura, M., Pitts, L., Vilnis, V., Tuerk, K., Lagger, R.: The role of ischemia in the pathogenesis of cervical spondylotic myelopathy: A review and new microangiographic evidence. Spine 1977; 2: 100-108.

31. Iseda, T., Goya, T., Nakano, S., Kodama, T., Moriyama, T., Wakisaka, S.: Serial changes in signal intensities of the adjacent discs on T2-weighted sagittal images after surgical treatment of cervical spondylosis: anterior interbody fusion versus expansive laminoplasty. Acta Neurochir (Wien.) 2001; 143: 707-710.

32. Jeffreys, R.V.: The surgical treatment of cervical myelopathy due to spondylosis and disc degeneration. J Neurol Neurosurg Psychiatry 1986; 49: 353-361.

33. Kadanka, Z., Bednarik, J., Vohanka, S., Vlach, O., Stejskal, L., Chaloupka, R., et al.: Conservative treatment versus surgery in spondylotic cervical myelopathy: a prospective randomised study. Eur. Spine J 2000; 9: 538-544.

34. Kawaguchi, Y., Matsui, H., Ishihara, H., Gejo, R., Yasuda, T.: Surgical outcome of cervical expansive laminoplasty in patients with diabetes mellitus. Spine 2000; 25: 551555.

35. Kohno, K., Kumon, Y., Oka, Y., Matsui, S., Ohue, S., Sakaki, S.: Evaluation of prognostic factors following expansive laminoplasty for cervical spinal stenotic myelopathy. Surg Neurol 1997; 48: 237-245.

36. Kohno, M., Takahashi, H., Yamakawa, K., Ide, K., Segawa, H.: Postoperative prognosis of Brown-Sequard-type myelopathy in patients with cervical lesions. Surg Neurol 1999; 51: 241-246.

37. Koyanagi, T., Hirabayashi, K., Satomi, K., Toyama, Y., Fujimura, Y.: Predictability of operative results of cervical compression myelopathy based on preoperative computed tomographic myelography. Spine 1993; 18: 1958-1963.

38. Kumar, V.G.R., Rea, G.L., Mervis, L.J., McGregor, J.M.: Cervical spondylotic myelopathy: functional and radiographic long-term outcome after laminectomy and posterior fusion. Neurosurgery 1999; 44: 771-778.

39. Law, M.D., Bernhardt, M., White, A.A.: Evaluation and management of cervical spondylotic myelopathy. Instr Course Lect 1995; 44: 99-110.

40. Lesoin, F., Bouasakao, N., Clarisse, J., Rousseaux, M., Jomin, M.: Results of surgical treatment of radiculomyelopathy caused by cervical arthrosis based on 1000 operations. Surg Neurol 1985; 23: 350-355.

41. Martín, R., Carda, J.R., Pinto J,I., Sanz, F., Montiaga, F., Paternina, B., et al.: Discectomía cervical y artrodesis intersomática por vía anterior mediante técnica de Cloward: análisis retrospectivo de las complicaciones y los resultados radiológicos en 167 casos. Neurocirugia 2002; 13: 265-284.

42. Martino, V., Nina, P., Franco, A., Di Benedetto, A., Chiappetta, F., Schisano, G.: Cervical myelopathy caused by median disc herniation: analysis of the complications follo- wing anterior discectomy with and without Fusion Report of 90 cases. J Neurosurg Sci 1997; 41: 153-58.

43. Matsuda, Y., Miyazaki, K., Tada, K., Yasuda, A., Nakayama, T., Murakami, H.. et al.: Increased MR signal intensity due to cervical myelopathy. Analysis of 29 surgical cases. Surg Radiol Anat. 1991; 74: 887-892.

44. Matsukado, Y., Yoshida, M., Goya, T., Shimoji, K.: Classification of cervical spondylosis or disc protrusion by preoperative evoked spinal electrogram. Follow-up study. Surg Radiol Anat. 1976; 44: 435-441.

45. Matsuyama, Y., Kawakami, N., Mimatsu, K.: Spinal cord expansion after decompression in cervical myelopathy. Investigation by computed tomography myelography and ultrasonography. Spine 1995; 20: 1657-1663.

46. Mehalic, T.F., Pezzuti, R.T., Applebaum, B.I.: Magnetic resonance imaging and cervical spondylotic myelopathy. Neurosurgery 1990; 26: 217-227.

47. Moore, A.P., Blumhardt, L.D.: A prospective survey of the causes of non-traumatic spastic paraparesis and tetraparesis in 585 patients. Spinal Cord 1997; 35: 361-367.

48. Morio, Y., Teshima, R., Nagashima, H., Nawata, K., Yamasaki, D., Nanjo, Y.: Correlation between operative outcomes of cervical compression myelopathy and mri of the spinal cord. Spine 2001; 26: 1238-1245.

49. Morio, Y., Yamamoto, K., Kuranobu, K., Murata, M., Tuda, K.: Does increased signal intensity of the spinal cord on MR images due to cervical myelopathy predict prognosis? Arch Orthop Trauma Surg 1994; 113: 254-259.

50. Naderi, S., Ozgen, S., Pamir, M.N., Ozek, M.M., Erzen, C.: Cervical spondylotic myelopathy: surgical results and factors affecting prognosis. Neurosurgery 1998; 43: 4350 .

51. Nagata, K., Ohashi, T., Abe, J., Morita, M., Inoue, A.: Cervical myelopathy in elderly patients: clinical results and MRI findings before and after decompression surgery. Spinal Cord 1996; 34: 220-226.

52. Nakamura, M., Fujimura, Y.: Magnetic resonance imaging of the spinal cord in cervical ossification of the posterior longitudinal ligament. Can it predict surgical outcome? Spine 1998; 23: 38-40.

53. Nurick, S.: The natural history and the results of surgical treatment of the spinal cord disorder associated with cervical spondylosis. Brain 1972; 95: 101-108.

54. Pérez-López, C., Isla, A., Álvarez, F., Sarmiento, M.A., García-Raya, P., Pérez-Álvarez, M.: Eficacia de la artrodesis en el abordaje posterior de la mielopatía cervical: estudio comparativo de una serie de 36 casos. Neurocirugía 2001; 12: 316-324.

55. Phillips, D.G.: Surgical treatment of myelopathy with cervical spondylosis. J Neurol Neurosurg Psychiatry 1973; 36: 879-884.

56. Restuccia, D., Di, L.V., Valeriani, M., Tonali, P., Mauguiere, F.: Segmental dysfunction of the cervical cord revealed 
by abnormalities of the spinal N13 potential in cervical spondylotic myelopathy. Neurology 1992; 42: 1054-1063.

57. Rowland, L.P.: Surgical treatment of cervical spondylotic myelopathy: time for a controlled trial. Neurology 1992; 42: 5-13.

58. Sampath, P., Bendebba, M., Davis, J.D., Ducker, T.B.: Outcome of patients treated for cervical myelopathy. A prospective, multicenter study with independent clinical review. Spine 2000; 25: 670-676.

59. Saunders, R.L.: Anterior reconstructive procedures in cervical spondylotic myelopathy. Clin Neurosurg 1991; 37 : 682-6721.

60. Saunders, R.L., Bernini, P.M., Shirreffs, T.G.J., Reeves, A.G.: Central corpectomy for cervical spondylotic myelopathy: a consecutive series with long-term follow-up evaluation. Surg Radiol Anat. 1991; 74: 163-170.

61. Selladurai, B.M.: Cervical myelopathy due to nuclear herniations in young adults: clinical and radiological profile, results of microdiscectomy without interbody fusion. J Neurol Neurosurg Psychiatry 1992; 55: 604-608.

62. Shinomiya, K., Okamoto, A., Komori, H., Matsuoka, T., Yoshida, H., Muto, N., et al.: Prognosticating study for cervical myelopathy using evoked spinal cord potentials. Spine 1990; 15: 1053-1057.

63. Shoda, E., Sumi, M., Kataoka, O., Mukai, H., Hurosaka, M.: Developmental and dynamic canal stenosis as radiological factors affecting surgical results of anterior cervical fusion for myelopathy. Spine 1999; 24: 1421-1424.

64. Singh, A., Crockard, H.A., Platts, A., Stevens, J.: Clinical and radiological correlates of severity and surgery-related outcome in cervical spondylosis. Surg Radiol Anat. 2001; 94: 189-198.

65. Takahashi, M., Sakamoto, Y., Miyawaki, M., Bussaka, H.: Increased MR signal intensity secondary to chronic cervical cord compression. Neuroradiology 1987; 29: 550-556.

66. Tanaka, J., Seki, N., Tokimura, F., Doi, K., Inoue, S.: Operative results of canal-expansive laminoplasty for cervical spondylotic myelopathy in elderly patients. Spine 1999; 24 : 2308-2312.

67. Tani, T., Ushida, T., Yamamoto, H.: Surgical treatment guided by spinal cord evoked potentials for tetraparesis due to cervical spondylosis. Paraplegia 1995; 33: 354-358.

68. Taylor, A.R.: Vascular factors in the myelopathy associated with cervical spondylosis. Neurology 1964; 14: 62-68.
69. Tsiptsios, I., Fotiou, F., Sitzoglou, K., Fountoulakis, K.N.: Neurophysiological investigation of cervical spondylosis. Electromyogr Clin Neurophysiol 2001; 41: 305-313.

70. Wada, E., Ohmura, M., Yonenobu, K.: Intramedullary changes of the spinal cord in cervical spondylotic myelopathy. Spine 1995; 20: 2232.

71. Wada, E., Suzuki, S., Kanazawa, A., Mastsuoka, T., Miyamoto, S., Yonenobu, K.: Subtotal corpectomy versus laminoplasty for multilevel cervical spondylotic myelopathy. A long-term follow-up study over 10 years. Spine 2001; 26: 1443-1448.

72. Wada, E., Yonenobu, K., Suzuki, S., Kanazawa, A., Ochi, T.: Can intramedullary signal change on magnetic resonance imaging predict surgical outcome in cervical spondylotic myelopathy? Spine 1999; 24: 455-461.

73. Wohlert, L., Buhl, M., Eriksen, E.F., Fode, K., Klaerke, A., Kroyer, L., et al.: Treatment of cervical disc disease using Cloward's technique. III. Evaluation of cervical spondylotic myelopathy in 138 cases. Acta Neurochir (Wien.) 1984; 71: 121-131.

74. Yonenobu, K., Abumi, K., Nagata, K., Taketomi, E., Ueyama, K.: Interobserver and intraobserver reliability of the japanese orthopaedic association scoring system for evaluation of cervical compression myelopathy. Spine 2001; 26 : 1890-1894.

75. Yonenobu, K., Hosono, N., Iwasaki, M., Asano, M., Ono, K.: Laminoplasty versus subtotal corpectomy. A comparative study of results in multisegmental cervical spondylotic myelopathy. Spine 1992; 17: 1281-1284.

76. Yonenobu, K., Okada, K., Fuji, T., Fujiwara, K., Yamashita, K., Ono, K.: Causes of neurologic deterioration following surgical treatment of cervical myelopathy. Spine 1986; 11: 818-823.

Martín, R.; Carda, J.F.; Montiaga, F.; Pinto, J.I.; Sanz, F.; Paternina, B.; Trigueros, F.; Izquierdo, J.M.; Vázquez-Barquero, A.: Mielopatía cervical: análisis retrospectivo de los resultados quirúrgicos de 54 pacientes tratados mediante discectomía y fusión intersomática por vía anterior. Neurocirugía 2005; 16: 235-255.

Correspondencia postal: Rubén Martín Láez. Servicio de Neurocirugía. Hospital Universitario "Marqués de Valdecilla". Avenida Marqués de Valdecilla s/n. 39008 Santander 